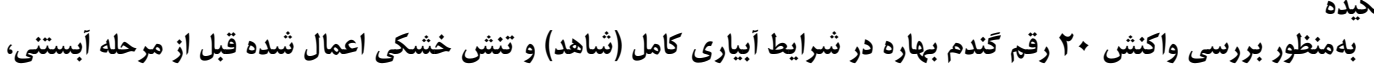

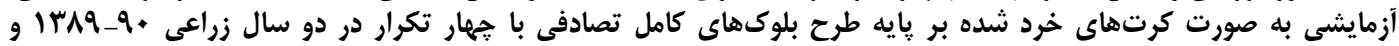

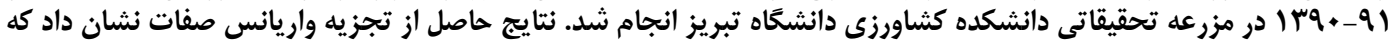

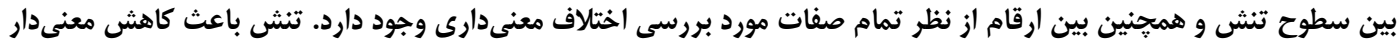

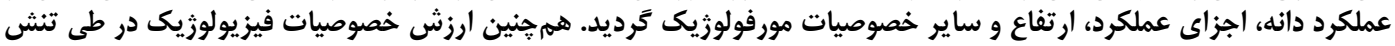

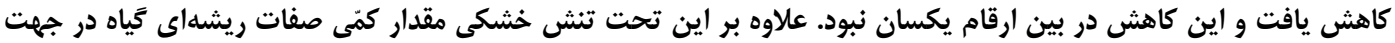

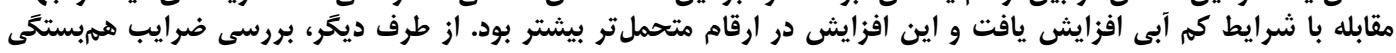

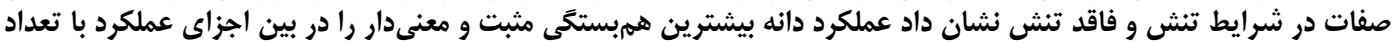

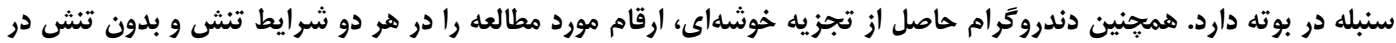

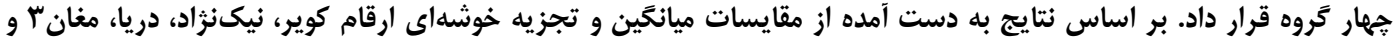

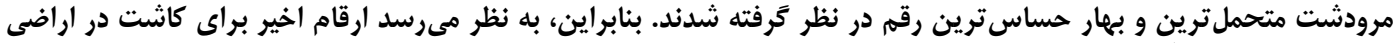

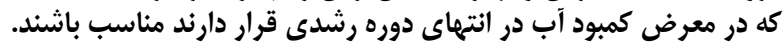

وازههاى كليدى: تجزيه خوشهاى، تحمل تنش، تنش خشكى، كَندم بهاره

مورد اشاره قرار گرفته است. سينگ و همكاران (F)

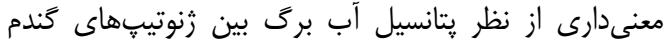

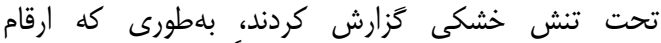

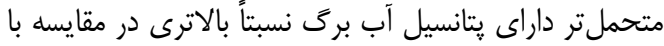

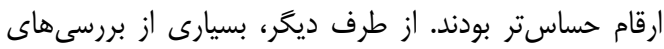

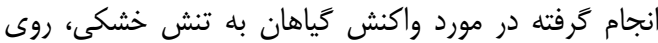

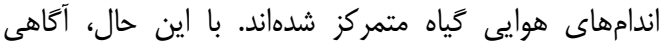

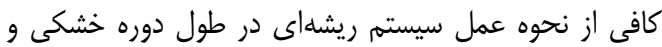

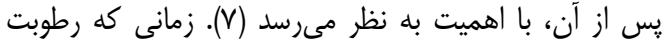

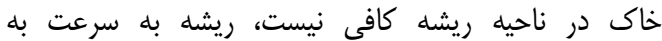

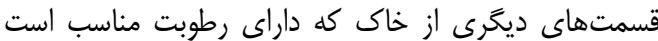

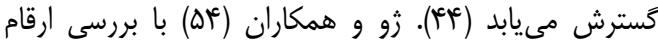

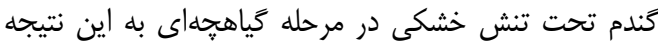

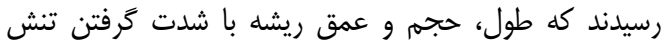

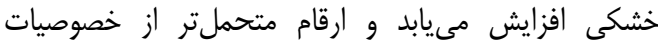

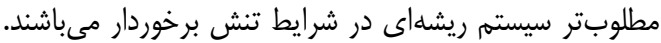

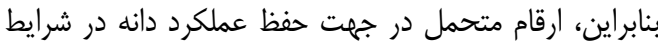

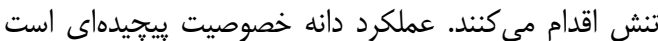

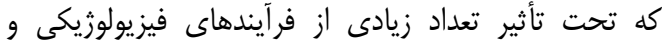

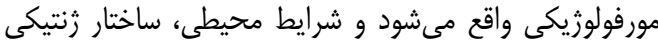

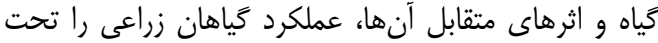

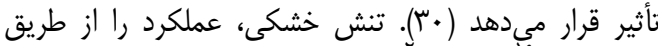

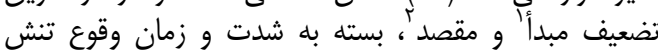

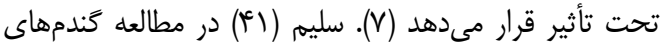

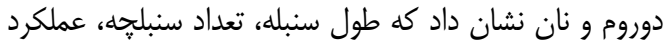
دانه، ماده خشك بوته و ارتفاع بوته در شرايط تنش دن خشكى نشى

1- Source
تنش خشكى يكى از علل اصلى خسارت به گياهان زراعى

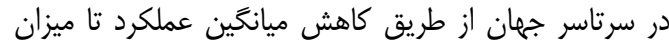

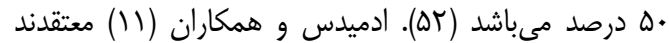

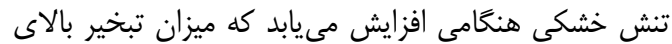

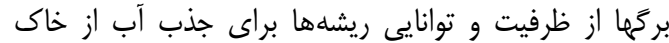

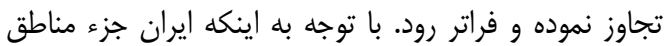

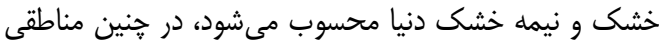

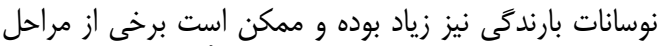

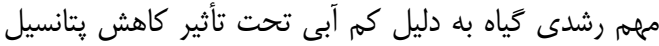

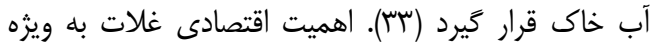

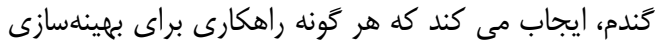

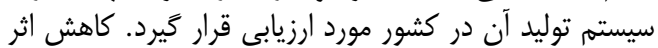

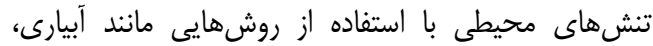

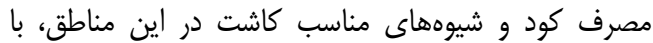

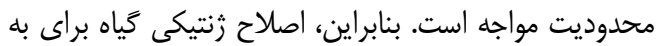

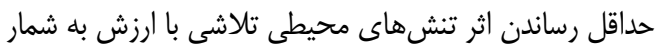

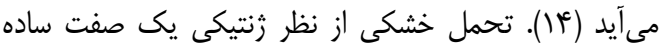

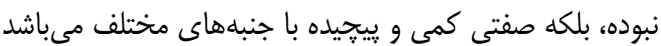

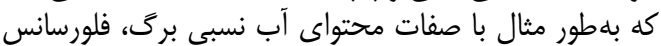

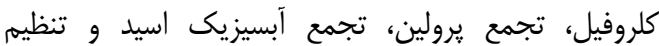

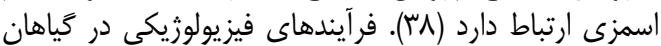

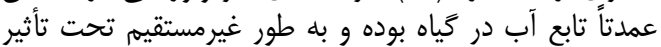

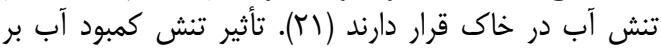

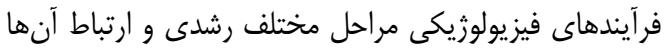

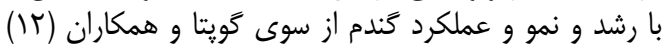




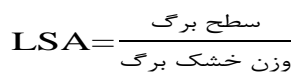

(r) رابطه

يتانسيل اسمزى با استفاده از دستخاه اسمومتر مدل (Osmomat o10, Gonotec) اندازهگيرى شد. فلورسانس كلروفيل: براى اين كار از دستخاه فلورومتر

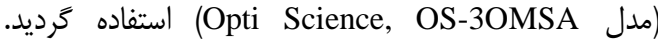
شاخص كلروفيل برگها با استفاده از دستخاه كلروفيلمتر

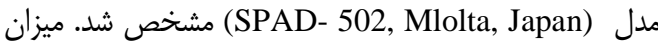

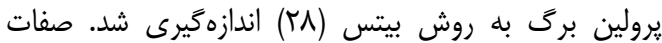

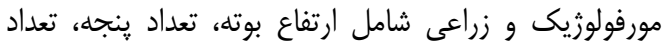

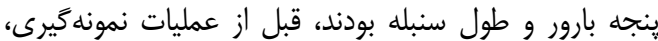

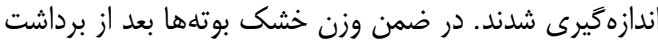

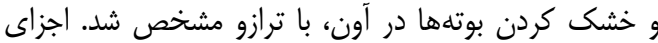

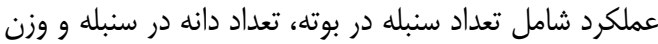

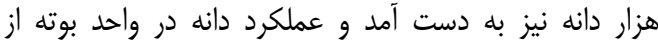
Y رابطه (r) a : تعداد سنبله در بوته، b: تعداد دانه در سنبله وcc: وزن هزار دانه

تجزيه واريانس مركب دادهها براساس آزمايش كرتهاى

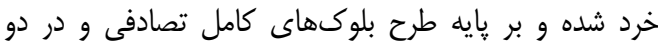

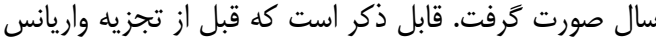

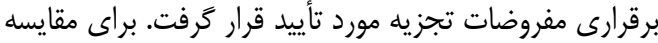

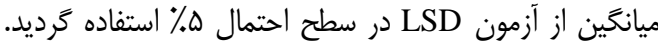

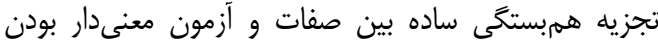

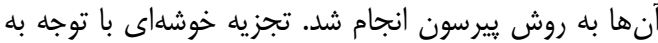

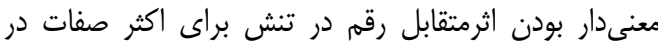

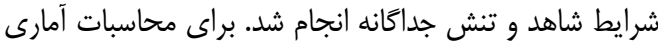

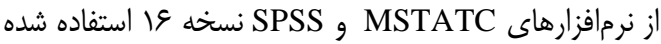
و براى رسم نمودارها از نرمافزار Excel استفاده شد.

تجزيه واريانس تفاوت معنى دانسارى بين ارقام براى كليه

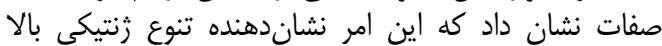

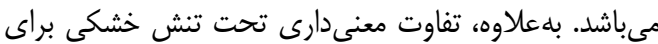

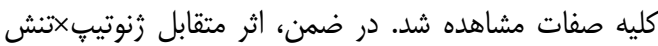

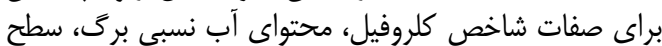

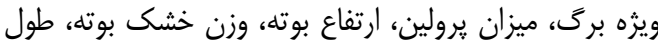

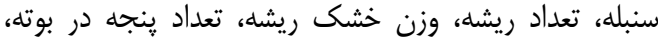

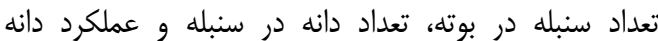

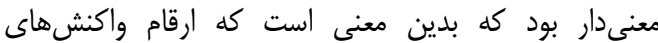

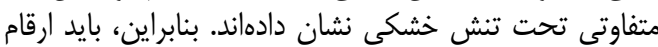

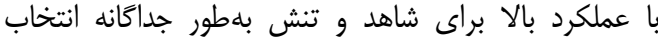

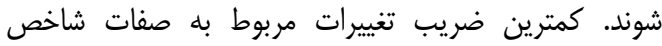

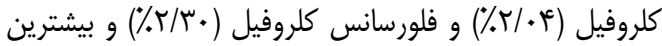

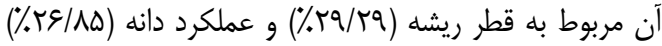
بود. به نظر مىرسد كه قطر ريشه و و عملكرد دانه بيشتر از
كاهش مىيابد. بنابراين، با توجه به اهميت كَندم و تنش إنش

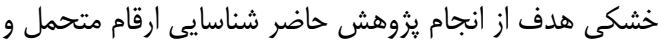

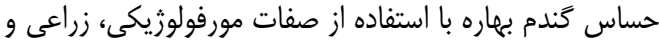

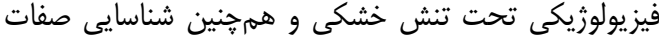
مطلوب جهت زَزينش ارقام متحمل مى بـ باشد.

\section{مواد و روشهات}

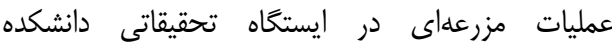

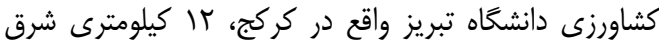

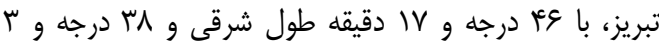

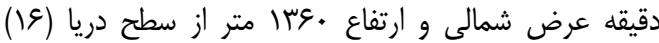

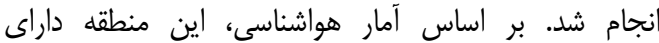

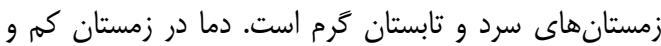

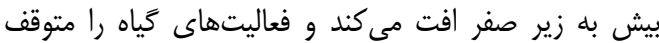

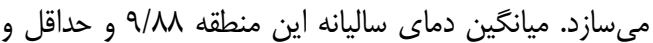

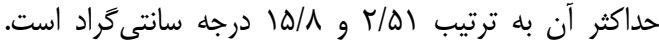

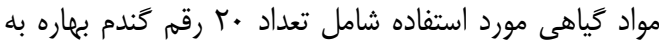

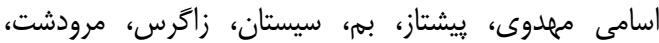

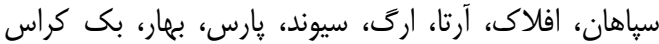

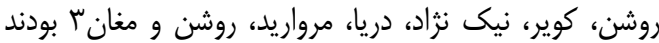

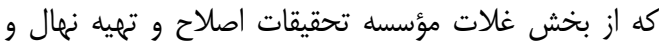

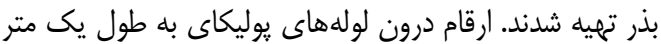

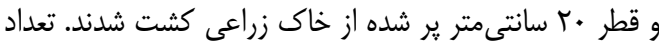

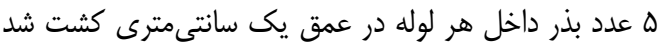

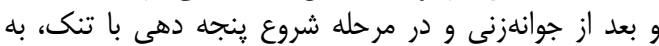

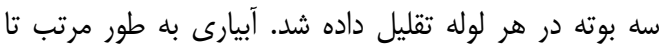

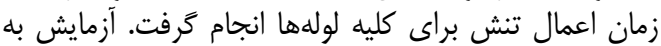

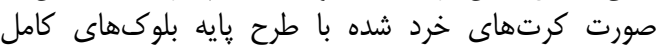

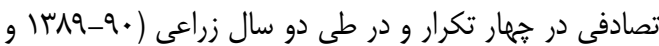

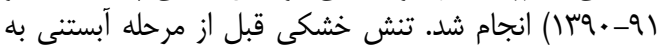

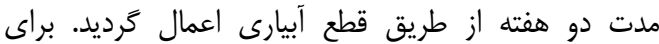

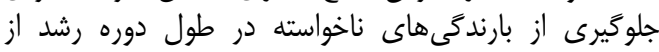

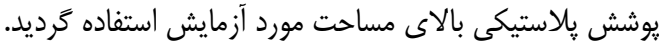

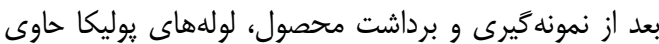

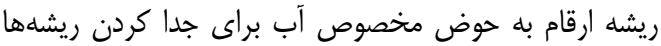

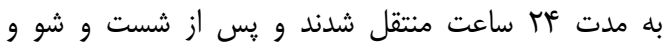

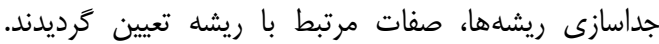

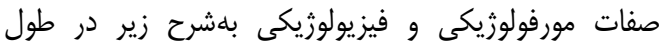

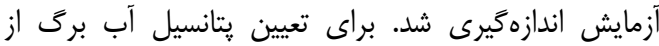

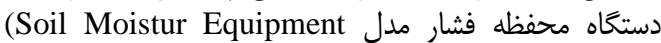
crop, Sanat Barbara, CA) با استفاده از دماسنج مادون قرمز مشخص شد. محتواى آب

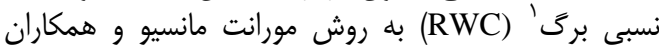

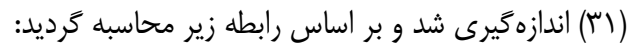

رWC

سطح ويثه برَّ (LSA) ) از طريق محاسبه نسبت سطح

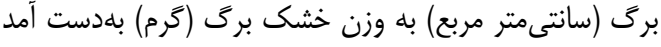




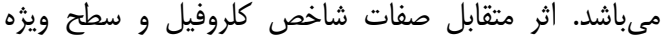

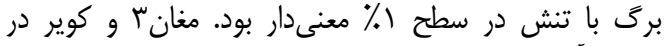

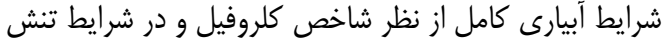

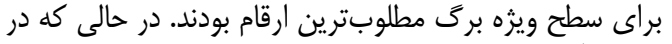

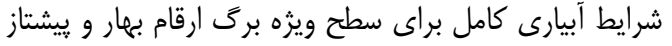

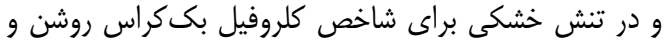

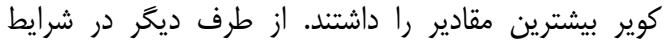

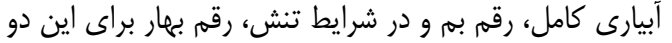

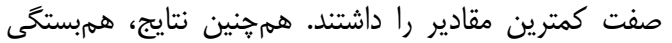

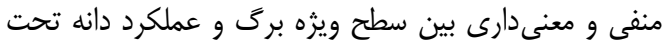

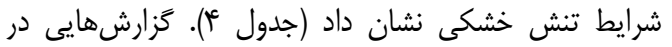

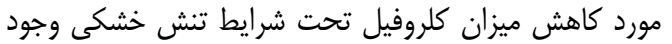

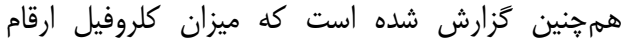

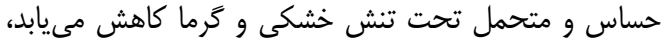

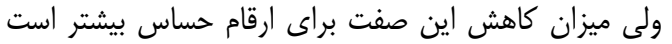

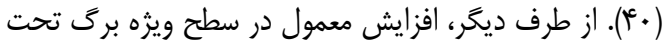
شرايط تنش خشكى ممكن است ناشى از از از دست دادئ دادن وزئن

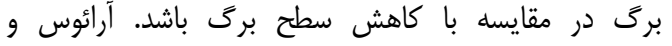

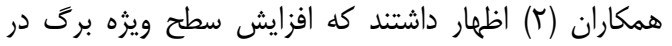
تنش خشكى ممكن است، در اثر سازكارى با با شرايط تنش

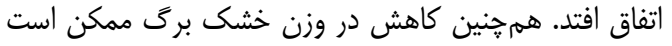

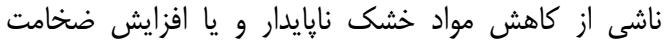

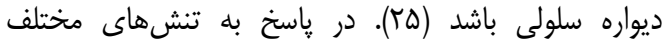

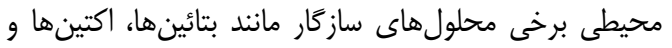

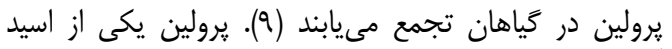

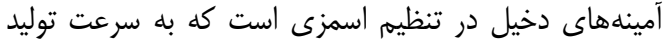

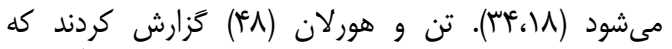

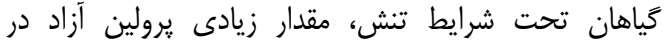

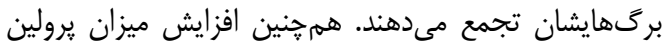

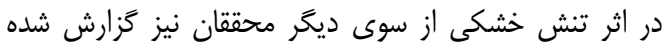

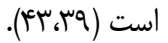

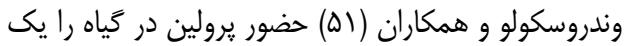

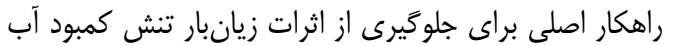

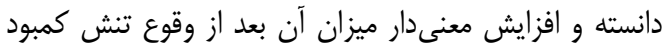

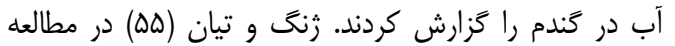

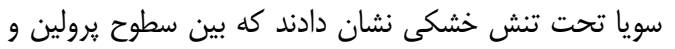

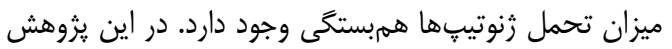

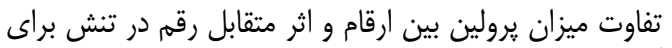

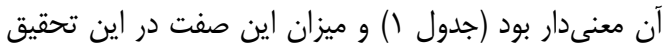

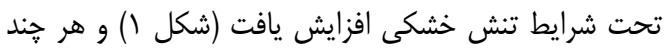

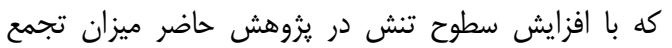

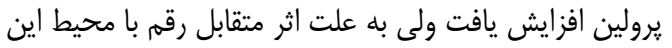

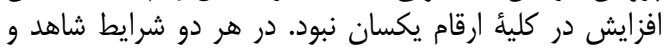

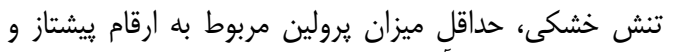

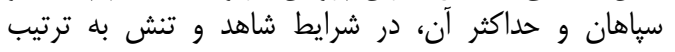

مربوط به ارقام مغان سّ و كوير، و بهار و كوير بود (شكل ().
ساير صفات تحت تأثير محيط قرار مى كَيرند. افزون بر اينها

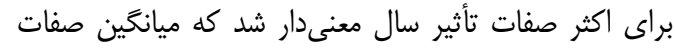

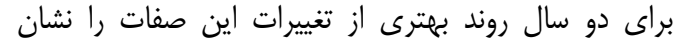

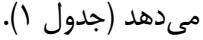

مقايسات ميانگين

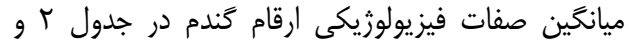

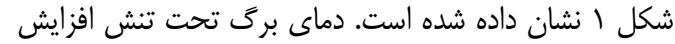

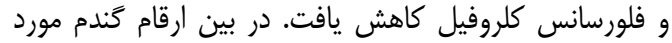

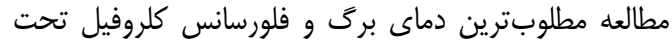

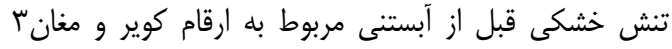

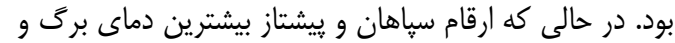

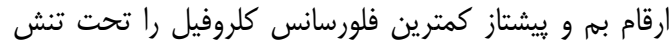

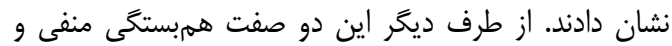

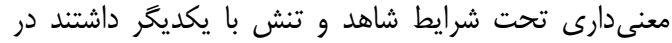

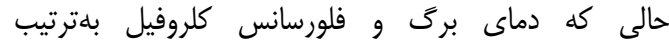

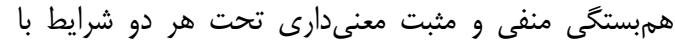

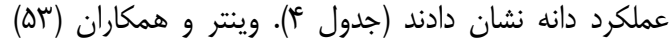

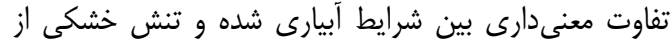

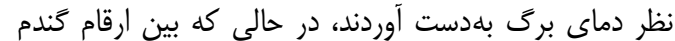

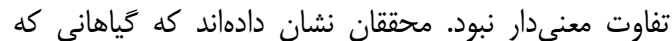

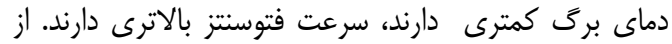

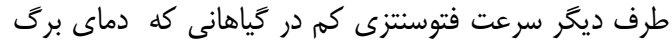

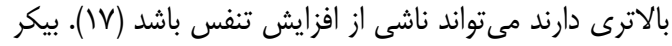

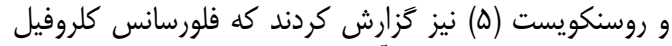

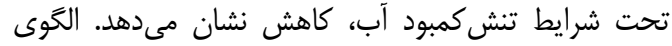
تغيير فلورسانس كلروفيل مشاهده شده در در اين مطالعن مشان مشابه

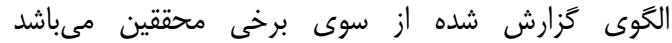

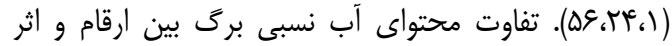

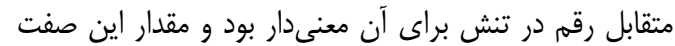

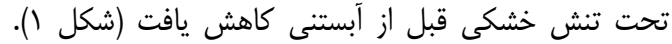

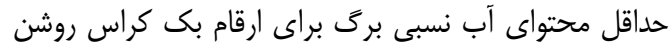

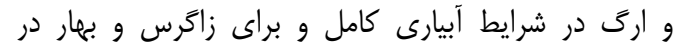

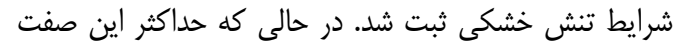

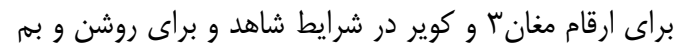

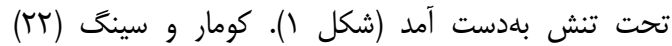

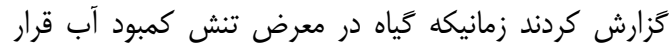

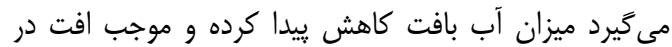

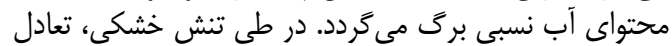

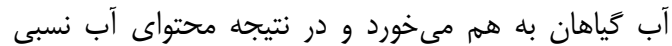

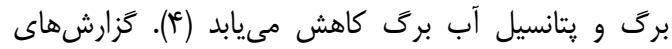

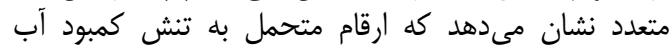

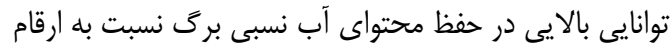

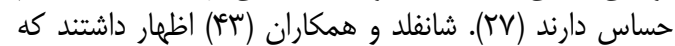

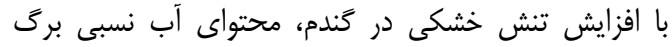

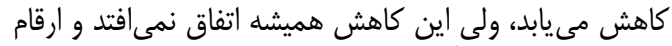

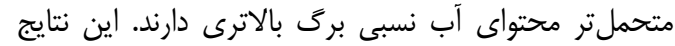

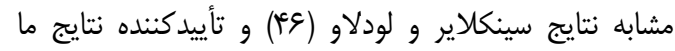


جدول ا- تجزيه مركب صفات مورد مطالعه كندم بهاره در شرايط تنش خشكى قبل از آبستى

Table 1. Combined analysis of the studied traits of spring wheat under drought stress before booting stage

\begin{tabular}{|c|c|c|c|c|c|c|c|c|c|c|c|c|}
\hline \multicolumn{11}{|c|}{ ميانكين مربعات } & \multirow[b]{2}{*}{ درجه آزادى } & \multirow[b]{2}{*}{ منابع تغييرات } \\
\hline طول سنبله & وزن خشك بوته & ار ارتفاع بوته & پِتانسيل اسمزى & يتانسيل كل آب & ميزان برولين & سطح ويره برى & محتواى آب برى & شاخص كلروفيل & فلور سانس & دماى برى & & \\
\hline$v a / r \cdot r^{* * *}$ & $T V \cdot / F \varphi D^{* * *}$ & FTYN/9DQ** & $\cdot / \Delta W W^{* * *}$ & $V / \Lambda F f^{* * *}$ & 1/qAr** & $\cdot|\ldots \cdots+|$ & KEYG/rG"N*** & $r \psi \cdot / r^{* * *}$ &.$/ .99^{* *}$ & TAI/rQ ${ }^{* * *}$ & 1 & سال \\
\hline.$/ \Delta V$ & . 1 va & 年/ &.$/ . .1$ &.$/ . .1$ & $.1 . .9$ & N/9/f & - /TVE & $\cdot / r \cdot r$ & $1 /+4 \times 10^{-\infty}$ & 1/9DT & 8 & تكرار/ سال \\
\hline$r q 9 / \Delta \varphi^{* * *}$ & 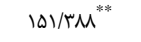 & $r \mid q \cdot V / r f^{* * *}$ & $I V / v \Lambda^{* *}$ & $\mathrm{IV} / \mathrm{IV} \mathrm{V}^{* * *}$ & $\Gamma /\left.\mu \Lambda\right|^{* *}$ & $11 . . / 94 r^{* * *}$ & $I V \Phi / V^{* * *}$ & $v \cdot 10^{* *}$ & $.1 .91^{* *}$ & $\mid r \varepsilon \cdot / \cdot v \Lambda^{* * *}$ & 1 & تنش \\
\hline.$/ . r$ & $1 / 91$ & זr./. & $V|\wedge| \times \mid)^{-9}$ &.$/ . .1$ & . $/ \ldots r \cdot r$ &.$|\ldots \cdots+|$ & $r / \cdot \vee V$ & $r / \% r$ & $81 \cdot+4 \times 1)^{-\Delta}$ & $T / F D$ & 1 & سال × تنش \\
\hline.$/ .11$ & . IN & .1059 &...$r$ &.$/ . r$ & .1 .19 & ११/१.V &.$/ N{ }^{*} \Lambda$ & אוז/. & $1 / 49 \times 1)^{-\Delta}$ & . IVGF & 9 & خطا \\
\hline $19 / \pi v^{* * *}$ & W/Arr*** & $T V g / T \cdot g^{* * *}$ &.$/ \pi v^{* *}$ &.$/ \Delta \Delta F^{* *}$ & . $/ 9\left\ulcorner 8^{* * *}\right.$ & $r q q \cdot / r \gamma e^{* * *}$ & rTr/Tr & $F / \epsilon q f^{* *}$ &.$/ .\left.\right|^{* * *}$ & $\mathrm{~V} / \mathrm{TV} \mathrm{V}^{* * *}$ & 19 & رقم \\
\hline $1 / . .9^{* * *}$ & . RVG & $9 / A V I$ &.$/ \% V$ & $\cdot|r|$ & $.1 .9 \Lambda^{* *}$ &.$|\ldots \ldots|$, & $|r / N|$ & $1 / e^{* * *}$ & . & $1 / 49$ & 19 & رقم × سال \\
\hline$\cdot|\Delta|^{*}$ & $r / \% q T^{* * *}$ & $\Delta V / \leftarrow \wedge)^{*}$ & $\%$ &.$/ \cdot 14$ & . $/ \Gamma \wedge \Delta^{* *}$ & TMTI/\&90** & $r q / \backslash \Lambda)^{* *}$ & $r / A q^{* *}$ & . & $r / .91$ & 19 & رقمم × تنش \\
\hline .1 .9 & r & $\mathrm{V} / \bar{g} \mathrm{~V}$ & .1 .11 &.$/ 14$ & .1 .14 & $\cdot|\ldots \ldots \cdot|$ & S/GMT &.$/ 9 \Delta 8$ & $9 / 49 \times 10^{-\Delta}$ & ג גז/. & 19 & رقم × تنش × سال \\
\hline$\cdot / r \cdot F$ & 1/TrE & $r q / r v q$ & .1 .91 & $.1 .+\mathrm{v}$ & .1 .19 & $10 Q / F A F$ & $9 / .1 \mathrm{~V}$ &.$/ 999$ & . $\ldots r V$ & $T / T V$ & TrA & خطا \\
\hline $9 / r$. & $N \cdot F$ & $11 / 94$ & $19 / \Delta 9$ & $1 . / \mathrm{Vg}$ & $r / q \pi$ & $V / 99$ & $r / T$ & $T / \cdot F$ & $r / \%$. & $\Delta / \bar{\Delta}$. & & ضريب تغييرات (٪) \\
\hline
\end{tabular}

ترتي معنى دارى در سطح احتمال هـ \% 1\% مىباشد

ادامه جدول 1

Table 1. continued

\begin{tabular}{|c|c|c|c|c|c|c|c|c|c|c|c|c|}
\hline \multirow[b]{2}{*}{ عملكرد دانه } & \multirow[b]{2}{*}{ وزن هزار دانه } & \multicolumn{9}{|c|}{ ميانكَين مربعات } & \multirow[b]{2}{*}{ درجه آزادى } & \multirow[b]{2}{*}{ منابع تغييرات } \\
\hline & & تعداد دانه در & تعداد سنبله در & تعداد ينجه باور & تعداد ينجه در & قطر ريشه & وزن خشك ريشه & حجم ريشه & طول ريشه & تعداد ريشه در بوته & & \\
\hline .1 .90 & $r / N^{c} \mid$ &.$|\ldots \ldots \cdot|$ & $|x| \cdot \cdot^{-y}$ & $r q / r^{* * *}$ & $F Y / \cdot D^{* *}$ & r/QAr & NGY $I^{* * *}$ & $r \mid / r \Delta F^{* *}$ & $\Delta \cdot / M^{* * *}$ & .9 .4 & 1 & سال \\
\hline$r / .9 V$ & $I V / r V$ & $r r / r \Delta r^{*}$ & $r / \Delta F r^{*}$ & $\Delta / f^{* * *}$ & $\Delta / \& \Delta \Lambda^{* *}$ & $.1 . \mu$ & $. / r / 9$ &.$/ T r q$ & . $/ . r 4$ & $19 / \leftarrow \Delta \Delta$ & 8 & تكرار/ سال \\
\hline$M F \cdot F / \Delta S \psi^{* * *}$ & $94 \mathrm{~T} / 19 r^{* * *}$ & $r q \uparrow \cdot / r \mid r^{* * *}$ & $V \Psi \tau / \cdot \Delta^{* * *}$ & VSN/A** & $\mathrm{Vqr} / \mathrm{\Lambda}^{* *}$ & $W / r \Delta r^{* * *}$ & TAV $/ V \Delta \Lambda^{* * *}$ & М१९/qVr ${ }^{* * *}$ & r $\checkmark \wedge q / \Delta \cdot D^{* * *}$ & Irsrq/vVA** & 1 & تنش \\
\hline. $\mid 91 \mathrm{~V}$ & $9 / 891$ &.$/ .14$ & $|x|^{-y}$ &.$/ \% \Delta$ &.$/ F \Delta$ &.$/ \ldots 190$ &.$/ . r r$ & . &.$\cdots \Delta$ & . /OTA & 1 & سال × تنش \\
\hline $8 / \pi / 9$ & r./DTQ & V/AFs & $r / .+r$ & $r / v \cdot \Lambda$ & $r / 99 V^{*}$ &.$/ 4 r$ &.$/ . e^{c}$ &.$/ N F$ & $\cdot / 1 \cdot 1$ & $9 / 9 V$ & 8 & خطا \\
\hline$r V / F V F^{* * *}$ & $\Delta I / V \Delta F^{* *}$ & $q \mathrm{~N} / . . \mathrm{f}^{* * *}$ & $r \Delta / \Delta \Delta \Delta^{* *}$ & $r / q r^{* *}$ & $r \psi / \Delta \varphi^{* * *}$ &.$/ 8 \wedge q^{* *}$ & $V / \Lambda \Delta r^{* *}$ & 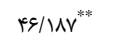 & $V \cdot / \Delta \Delta r^{* * *}$ & $9 \cdot / r \Delta \Gamma^{* * *}$ & 19 & رقم \\
\hline.$/ 999$ & $\mid T / \cdot \Lambda V$ & V/raV & $|x|^{-y}$ & V/DM & $1 / v \cdot 1$ &.$/ N F$ &. $\mid 8 \Lambda r^{*}$ & r/991 & S/TDS & $19 . / g V^{* * *}$ & 19 & رقم × سال \\
\hline $1 . / r r 1^{* *}$ & $|s| \cdot \Delta \Delta$ & q./vqr" & $r / \wedge q^{*}$ & $r / . \Delta V$ & $r / r r q^{*}$ &.$/ 1 F 9$ & $1 / 4 \cdot \Lambda^{* *}$ & r/NTY & $V / \& \Delta \Delta$ & $\mathrm{N} / \mathrm{N} \mathrm{V}^{*}$ & 19 & رقم × تنش \\
\hline 19"/. & $V / r \cdot r^{\prime}$ & $11 / r \cdot r$ & $\left.|x|\right|^{-y}$ & V/QVQ & $1 / \& 94$ & $.1 .9 \mathrm{~V}$ & $\cdot \mid \Delta F V$ & r/qTr & NWVA & $r T / V Q T^{* *}$ & 19 & رقم × تنش × سال \\
\hline$r / / M$ & $q / N \subset v$ & $14 / .94$ & I/gra & $1 / F N G$ & 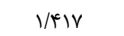 & . & 年 & Tr/r & $\Delta / \Delta T q$ & II/QUI & TMA & خطا \\
\hline$\Gamma \& / \Lambda \Delta$ & NIV & $I T / N e^{k}$ & $r Y / . r$ & $T r / T V$ & $N / F^{q}$ & $r q / r q$ & 1N/9F & $11 / 4$. & $N \cdot r$ & $14 / 79$ & & ضريب تغييرات (٪) \\
\hline
\end{tabular}


كردنى دارى كاهش مى يابد. تورجى و همكاران (\$q) نيز كزارش

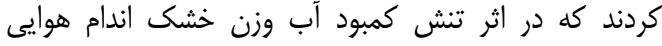

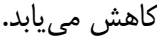
نتايج تجزيه واريانس نشان داد كه تفاوت معنى دارى بين

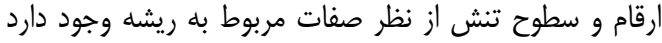

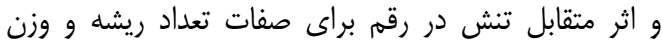

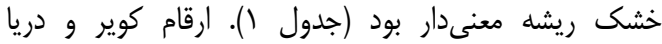

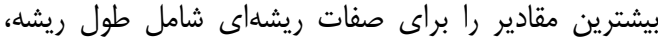

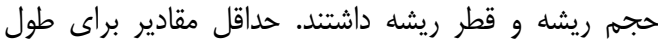

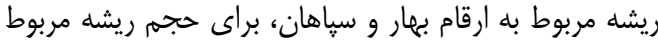

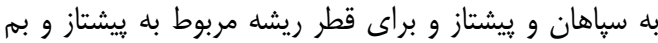

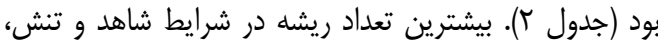

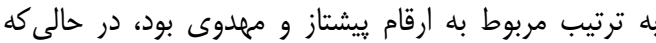

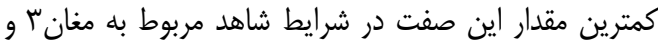

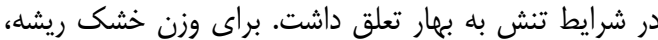

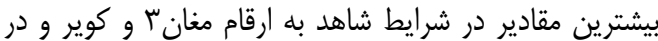

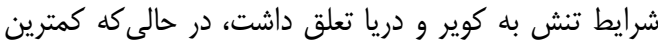

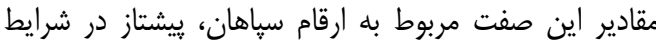

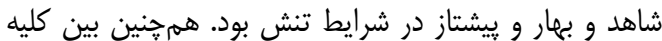

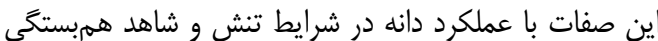

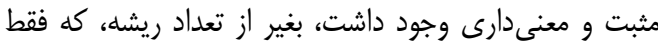

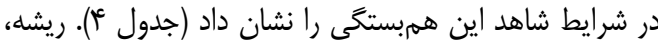

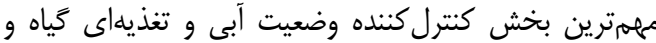

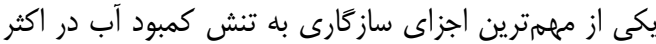

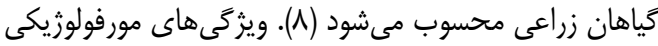

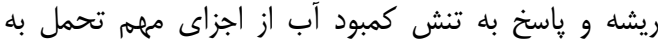

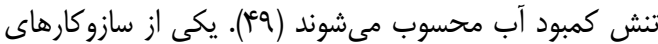

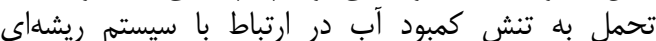
افزايش حداكثر طول ريشه براى استفاده بيشتر از ذخاير آبـ آبه

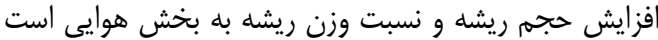

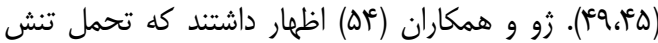

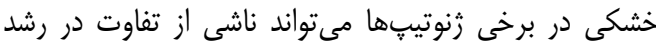

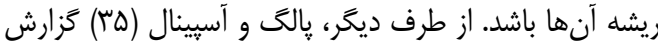

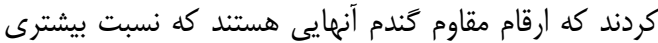

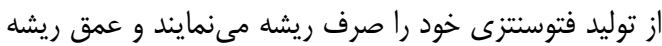

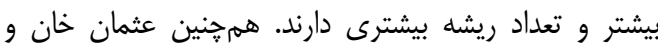

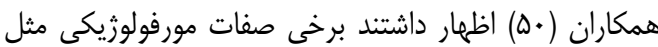

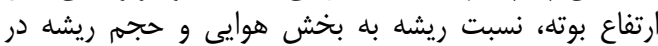

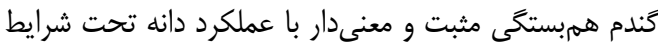

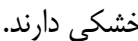

بر طبق جدول تجزيه واريانس تفاوت ارقام و سطوح تنش اري

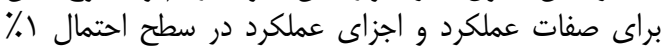

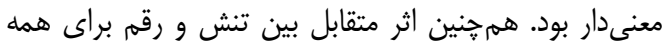

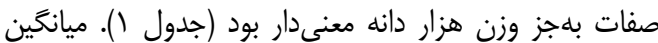

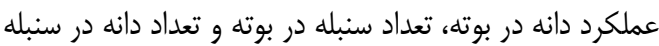

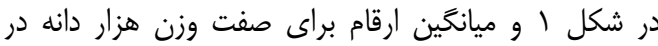

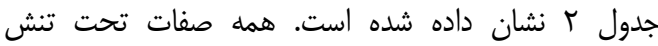

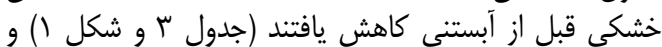
كاهش در تعداد دانه در سنبله بيشتر از كاهش وزئن إند هزار دانه
از طرف ديخر، تنش خشكى منجر به كاهش يتانسيل كل

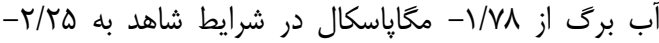

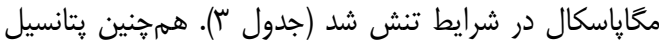

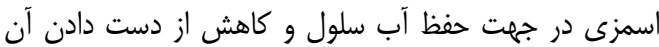

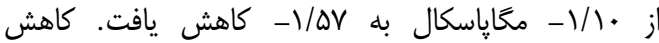

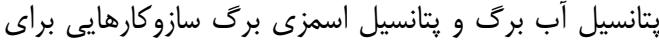

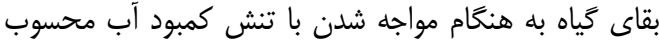

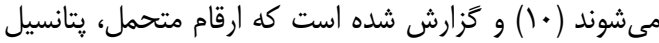

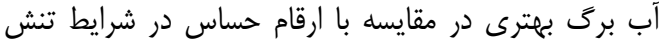

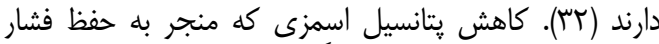

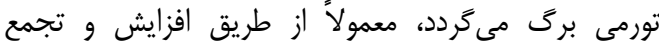

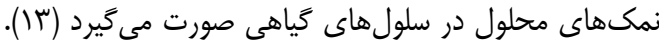

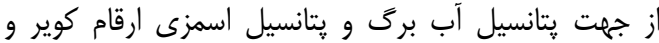

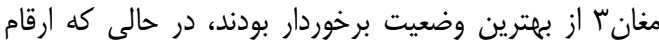

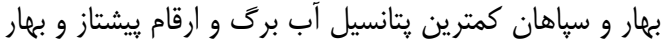

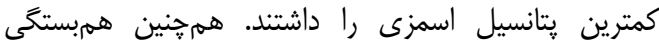

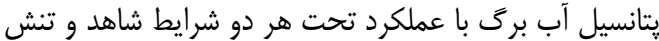

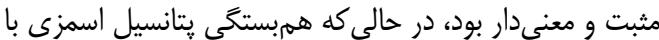

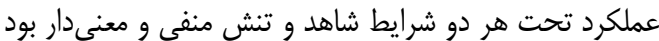

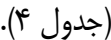

نتايج تجزيه واريانس تفاوت معنىدار بين ارقام كندم مورد

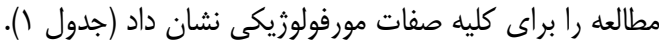

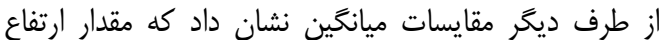

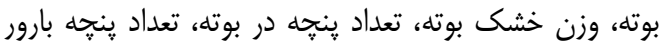

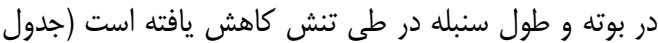

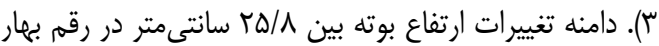

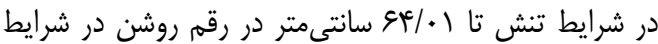

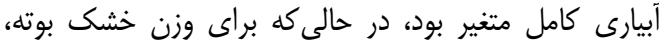

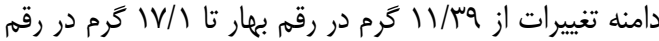

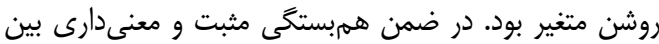

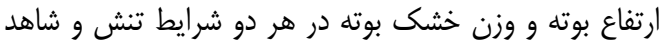

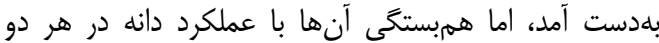

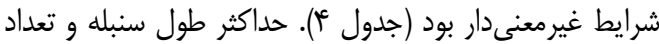

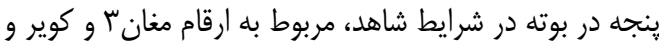

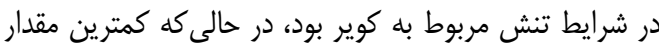

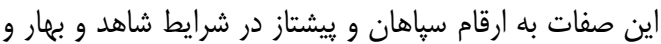

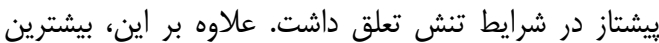

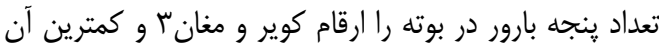

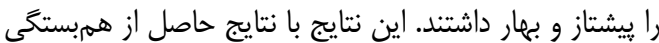

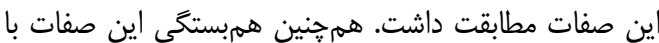

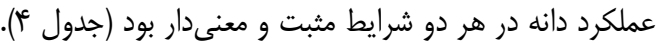

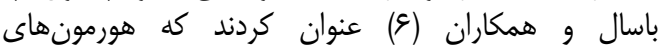
تنظيم كننده رشد نظير ABA در اندام هوايى در باسخ بان به به تنش

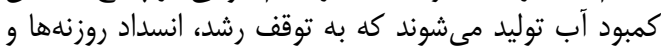

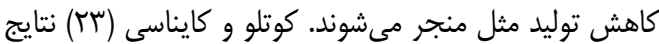

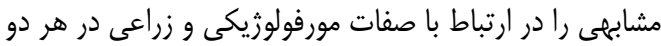

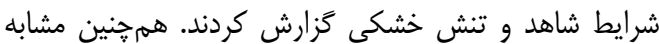

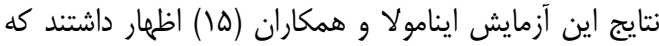

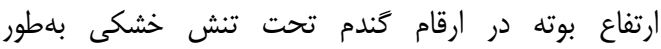


پِارس، زاگرس، آرتا و بم به خود اختصاص دادند و

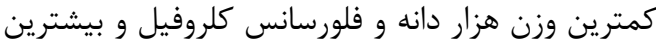

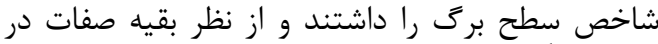

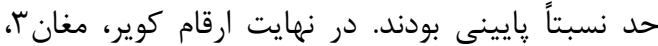

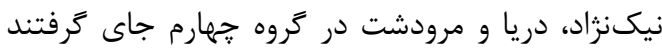

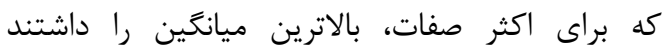

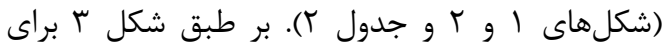

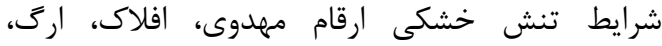

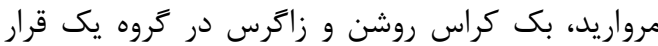

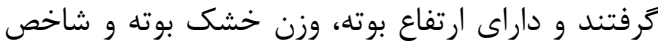

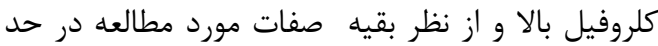

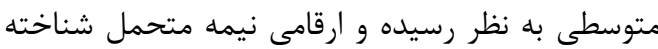

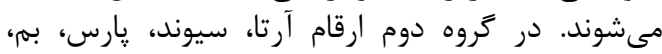

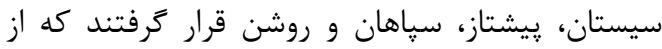

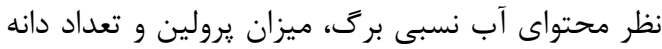

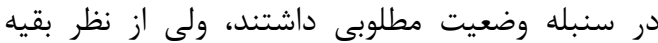

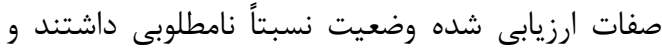

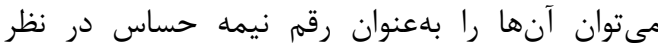

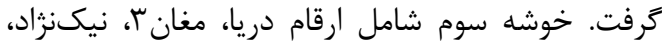

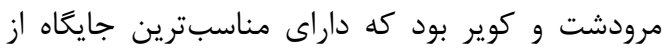

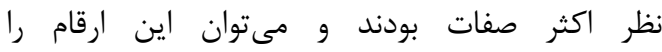

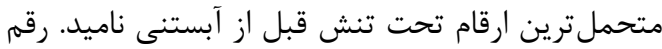
بهار نيز با حداقل ارزش كليه صفات ارجات بجز تبن تعداد دان دانه در

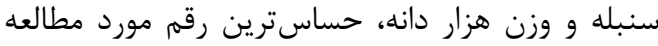

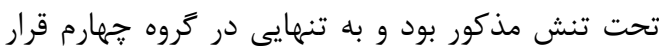

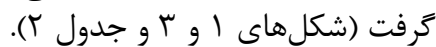

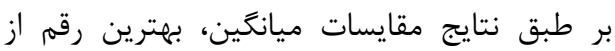

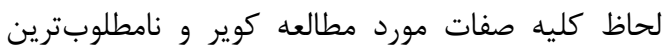

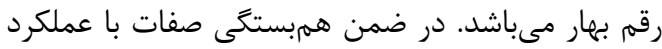

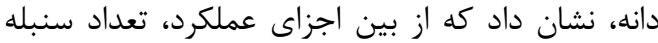

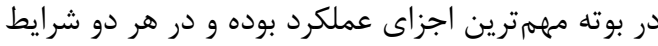

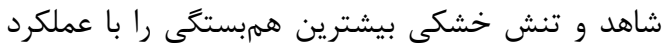

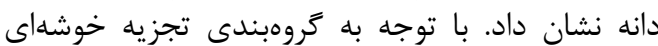

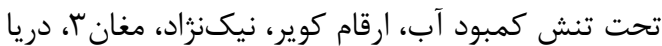

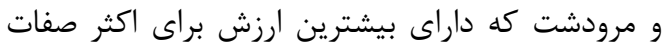

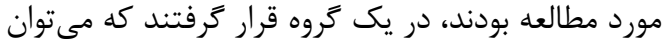

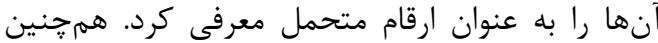

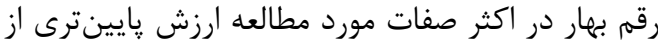

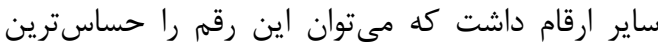

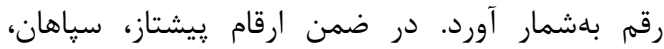

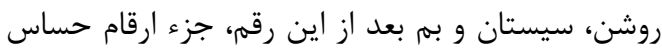

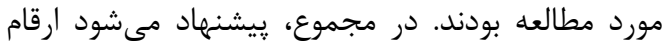

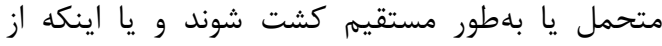
تلاقى اين ارقام با ارقام حساس تنوع جديدى ايجاد شود.
بود. همبستخى بين وزن هزار دانه و تعداد دانه در سنبله تنها

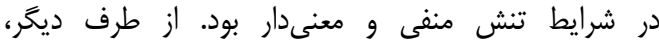

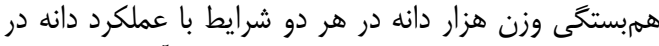

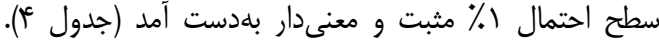

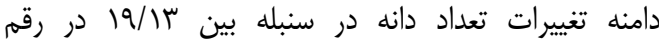

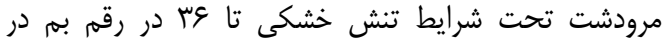

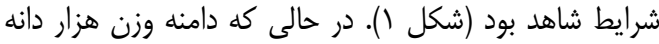

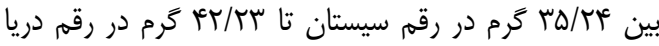

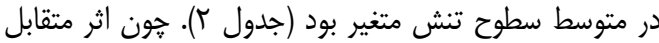

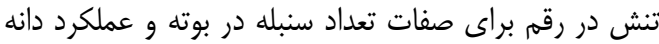

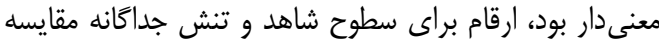

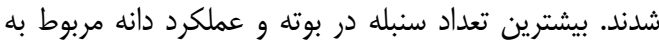

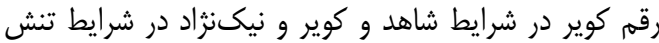

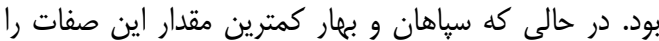

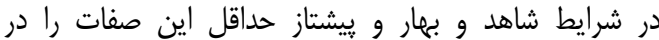

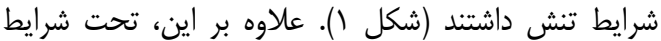

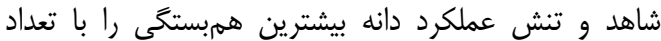

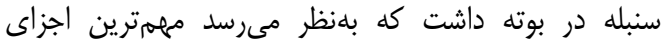

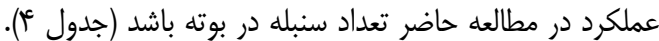

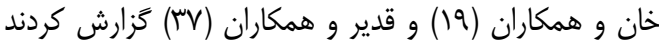

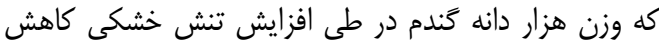

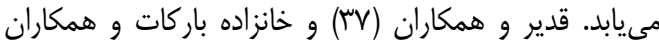

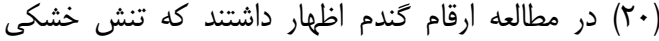

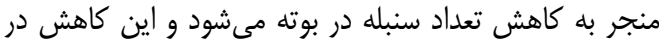

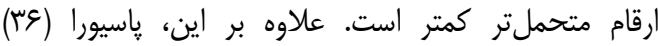

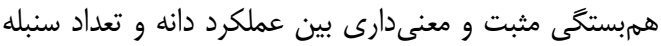

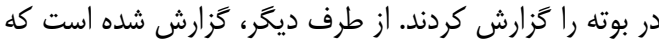

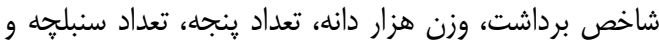

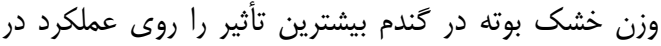

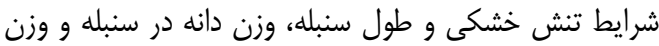

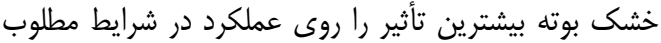

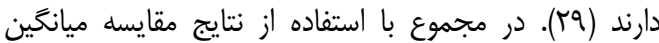

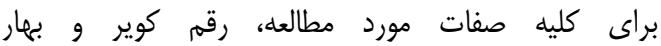

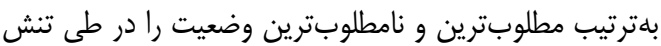
خشكى اعمال شده قبل از مرحله آبستنى داشتند.

تجزيه خوشهاى

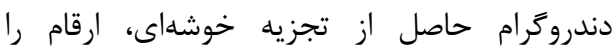

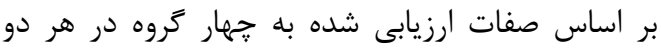
شرايط شاهد و تنش خشكى خلى تقسيم كرد.

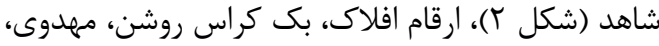

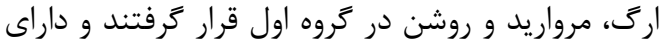

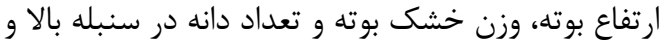

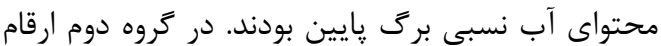

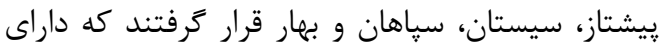

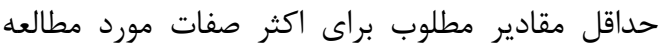
برگ بودند، در حالى كه مقادير شاخص سط سطح برى

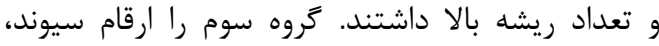


جدول r- مقايسه ميانخين ارقام گَندم بهاره از نظر صفات مورد مطالعه در متوسط سطوح تنش s. Table 2. Mean comparison of spring wheat cultivars for the studied traits in moderate of stress levels

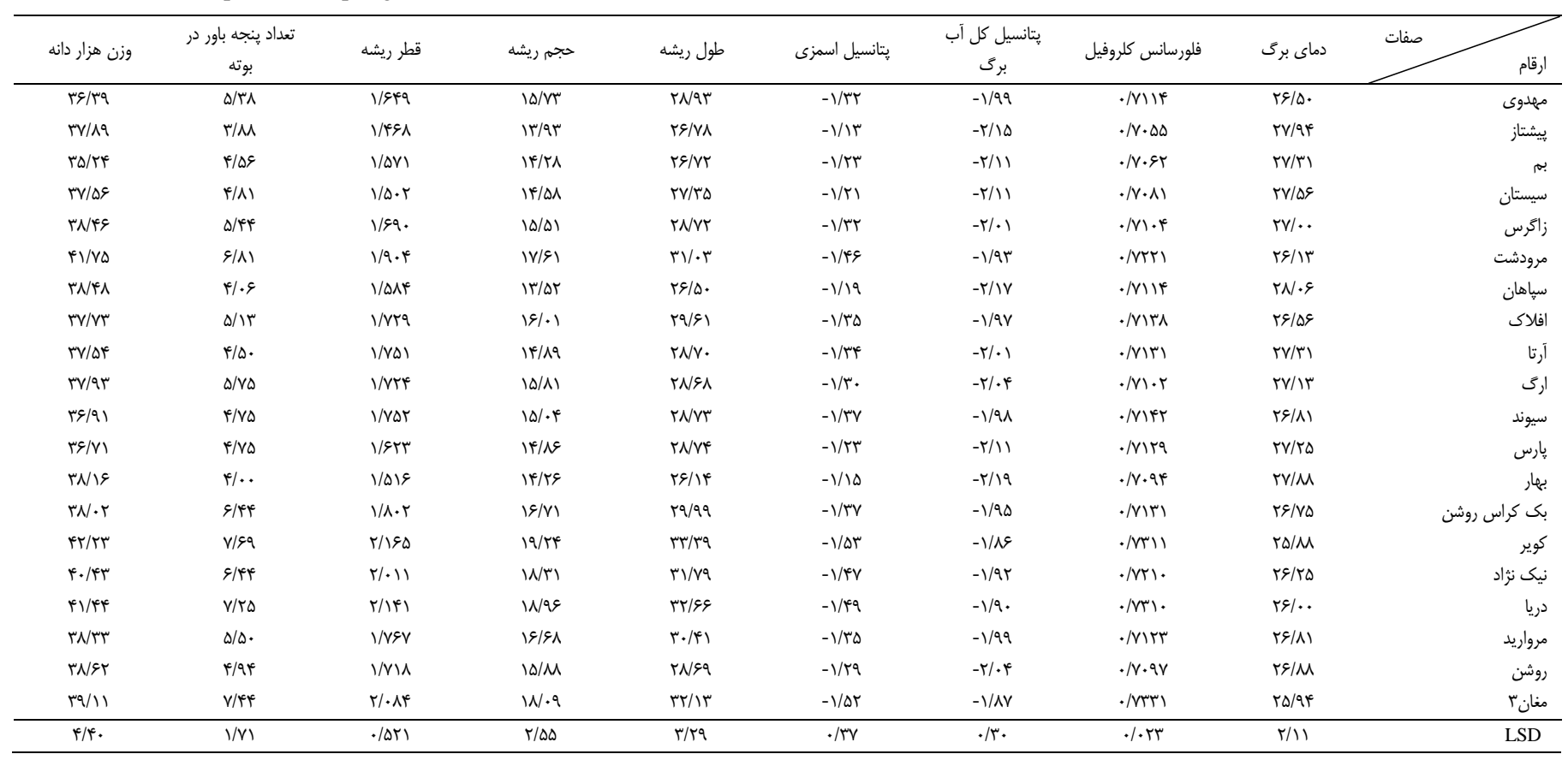

جدول ؟- مقايسه ميانگين سطوح تنش خشكى از نظر صفات مورد مطالعه

Table 3. Comparison the mean levels of drought stress for studied traits

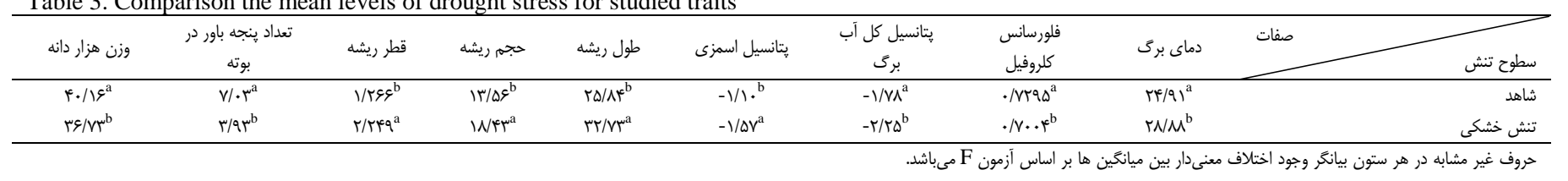



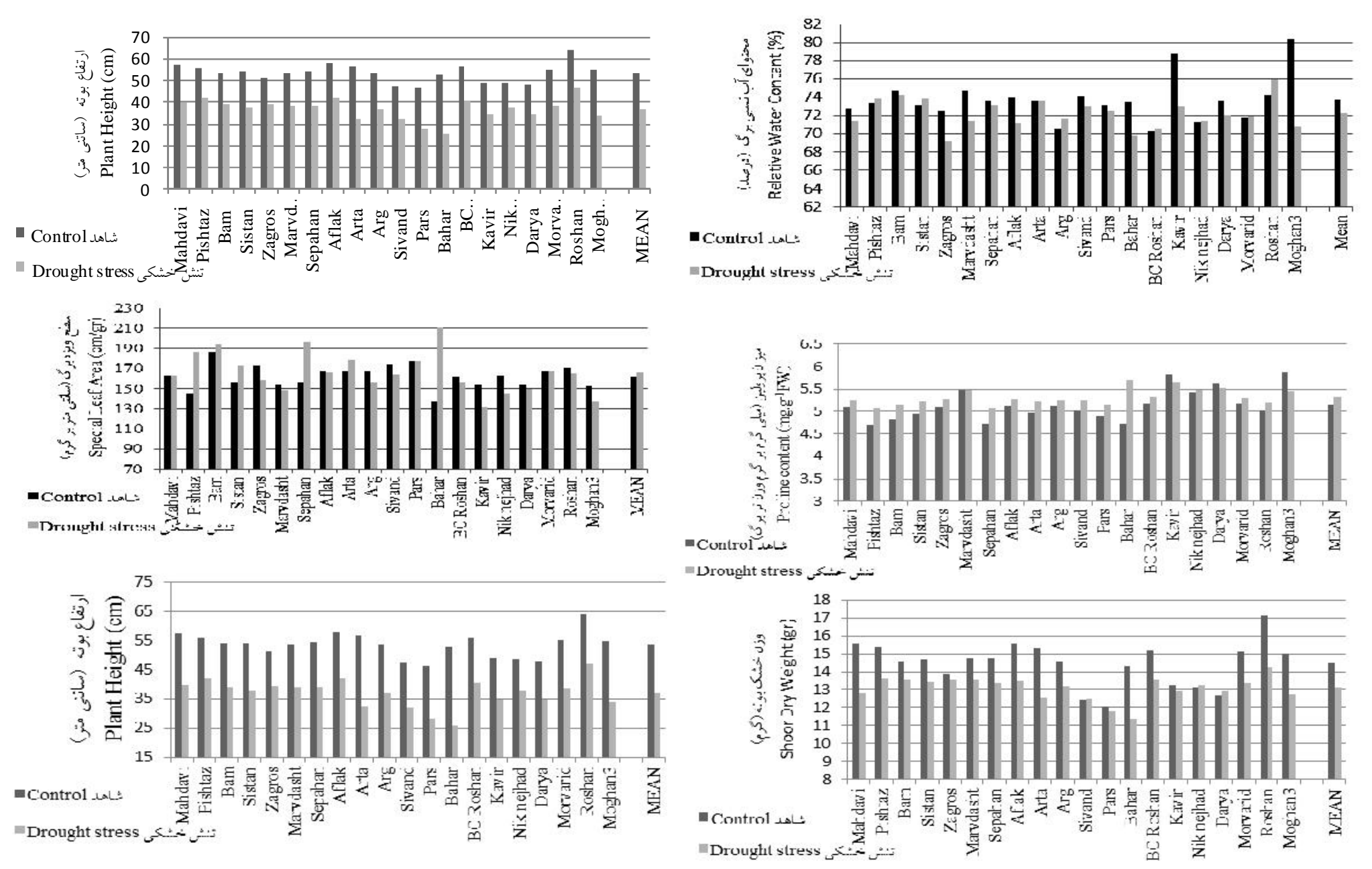

شكل ا- مقايسه ارقام گندم بهاره در صفات با اثرات متقابل تنش× رقم معنىدار در دو شرايط شاهد و تنش Figure1- Comparison of spring wheat cultivars for traits with significant effect of cultivar×stress under control and stress conditions 


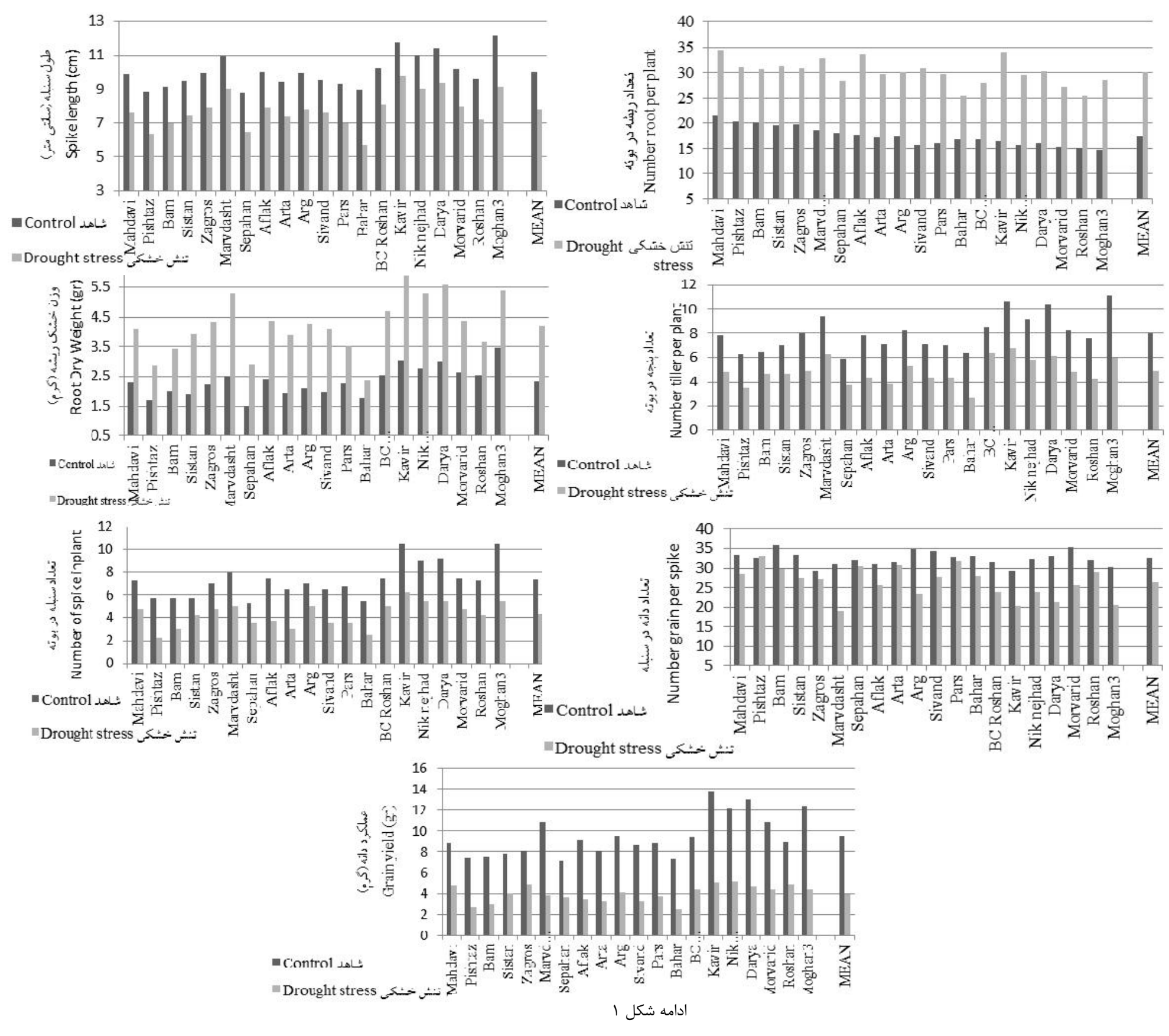


جدول ؟- همبستكى بين عملكرد دانه و ساير صفات مورد مطالعه كندم بهاره در شرايط شاهد (يايين قطر اصلى) و در شرايط تنش خشكى (بالاى قطر اصلى) Table 4. Correlation between grain yield and other studied traits of spring wheat in control conditions (below the main diagonal) and drought conditions (above the main diagonal)

\begin{tabular}{|c|c|c|c|c|c|c|c|c|c|c|c|c|c|c|c|c|c|c|c|c|c|c|}
\hline$\pi$ & $r$ & r. & 19 & in & iv & 18 & 10 & if & ir & Ir & 11 & 1. & 9 & $\wedge$ & r & 9 & $\Delta$ & r & r & r & 1 & صفات \\
\hline.$- \mid 9999^{* * *}$ &.$- / 59 q^{* 0 *}$ &. $\mid s p r r^{* *}$ & $. \cdot / N A^{* * *}$ & . $/ M r r^{* 0 *}$ & $-\cdot /$ var" & $-\cdot|\lambda r|^{* *}$ & $-/ / N y^{* *}$ &.$- /$ vaf $^{* *}$ & $-\cdot / A D^{* * *}$ &.$- / \Delta r a^{*}$ & $\cdot \cdot / \mathrm{MQ}^{* *}$ & 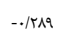 & $-. / \pi \mid s$ & $.19 .5^{* * *}$ & $-\cdot / A 9 F^{* *}$ &.$- / r \cdot 9$ & $\cdot \mid \mathrm{ASA}$ & $\cdot / 1 \Lambda$ &.$- / r A r$ & $\cdot / \Delta \Delta \gamma^{* *}$ & 1 & دماى برى \\
\hline.$/ 91.0^{* * *}$ & .Nreq" &.$- /$ /v9 & $\cdot(\Lambda \cdot)^{* * *}$ & $\cdot|\lambda r|^{* * *}$ & $\cdot / \mathrm{ArT}$ &.$/ 299^{* * *}$ & . $/$ arfe" &.$|a r|^{* *}$ & . & $\cdot / \mathrm{rr}$ &.$/ 499^{\circ "}$ &.$/{ }^{\prime}$ &.$- / . \cdot \mathrm{qa}$ &.$- /\left.94\right|^{* *}$ & . $/ a 1 r^{\prime \prime \prime}$ & . $\mid \Delta S r^{\prime \prime \prime}$ &.$- / A \Delta_{B}^{* *}$ & $. \cdot / 1 / N$ & . KFa & 1 & -.|sAr" & فلورسانس كلروفيل \\
\hline.$/ \% 4$ & 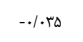 &.$- / / r r 4$ & . Frs & . $/ \mathrm{rra}$ &.$/ F F r$ &.$/ 10 r$ & $\mid k \& Y^{*}$ & $\cdot r \cdot v$ &.$/ \pi \%$ & $. / k+r$ & . & KSYY &.$/ F A F$ & $-. / r \mid r$ & . $/$ A. $^{*}$ &..$/ 1 r$. & $-\cdot / \Delta \cdot r^{*}$ &. .1 .54 & , & $\cdot \mid A r \Delta^{* * *}$ & -./v99"* & شاخص كلروفيل \\
\hline.$- / / T Y$ &.$- / 19$. &.$/ 4 \%$ & $-\cdot / r \cdot r$ &.$- /(T+1)$ & $-0 / \pi q q$ & $-\cdot / \Lambda$. & $-0 /\{9 \mathrm{~V}$ &.$- / 459$ & $-. / r+r$ & $-\cdot / \cdot v$ &.$- / \pi r r$ & F Fiv & $\cdot / \cdot \cdot \Delta$ & $\cdot r .$. &.$- / r$. &.$- / \Delta \cdot 1^{\circ}$ &.$/ \pi r$. & 1 & $\cdot / \mathrm{FrV}$ & $\mid\left(F+\left.\right|^{1 * *}\right.$ &.$- / \mathrm{rwr}$ & محتواى آب نسبى برك \\
\hline.$- / w v^{* * *}$ & $-\cdot(N)^{* *}$ & . $/ \operatorname{vr}^{* * *}$ &.$- / 9.5^{4 * *}$ & $\cdot \mid A+\Lambda^{* * *}$ & $-\cdot / A F^{\prime \prime}$ & $\cdot \cdot / \lambda r r^{* *}$ & $-. / 9.4 . "$ & $\cdot / / \wedge \Lambda^{* *}$ & $-\cdot / \mathrm{Arr}$ &.$- / r r r$ & $-. / a p r+"$ & 政/RS &.$- / 191$ & / $\operatorname{sir}$ &.$- / 495 y^{0 * *}$ &.$- / 4 n$ & 1 &.$- / \pi r r$ &.$- /$ rar & $-\cdot|\Delta| .^{*}$ & $\cdot \cdot \cdot r$ & سطح ويثرّ برى \\
\hline . $/ 2 \Delta \Delta$ & 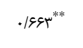 & $-\cdot / m f^{e *}$ & $.(\Delta))^{\circ}$ &. $\mid \xi \cdot r^{* * *}$ & . & . $18 \% \Lambda^{* * *}$ & $\cdot \mid \Delta t)^{*}$ & $\cdot N e^{* * *}$ & $\cdot / \triangle A Y V$ &.$/ . \Delta$ & $\left.\cdot|\Delta|^{*}\right|^{*}$ &.$- / F s y$ & $-. / 4 \mid s$ & $-\cdot / \Delta \mathrm{Ar} \sigma^{\circ}$ &.$/ 4 \lambda$ & 1 & $-\sim / \Lambda$. & $\cdot / \Delta \cdot \varphi^{\circ}$ & .apri" & $\cdot / \Lambda \mid V^{* *}$ & $\because / A A^{*}$ & ميزان يرولين \\
\hline $.199 \mathrm{~N}^{* * *}$ & $.1999^{* *}$ & $-\cdot / \mathrm{NeF}^{e *}$ & $\cdot|A|)^{* * *}$ & $\cdot \mid \mu \Lambda^{* * *}$ &.$M F^{*}$ & $\cdot / \mathrm{NV} \cdot{ }^{* *}$ &. $\mid a \Delta \sigma^{\circ "}$ & $\cdot / A v s^{* *}$ & $.19 .9^{\mathrm{*1}}$ & F ras & $.1900^{\circ *}$ & $\mid \pi N$ &.$/ 19$ &.$- / 94 \Delta \Delta^{* *}$ & 1 & $\cdot \mathrm{MM}^{\prime \prime}$ & $\cdots / . r$. & . /rav & . $/ 19 . " *$ & $.18 \Delta S^{* *}$ & $-\because / M q^{* \prime \prime}$ & يتانسيل كل آب برى \\
\hline.$- \mid 99 .^{* * *}$ & $-\cdot /$ Neq" & ./va"** & $\cdot \cdot / \mathrm{Arr}$ & $\cdot-{ }^{-}$ & -./Ns"*" &.$- / 90 s^{* \prime \prime}$ &.$- / 20 r^{\prime \prime *}$ &.$- / q r V^{* *}$ & -./ars" & $-\cdot / r \cdot 1$ &.$- / 9 F^{* *}$ & -./Mr &.$/ 11$ & 1 &.$- / 990^{\circ "}$ & $\cdot \cdot M r^{* *}$ &.$/ \cdot r$ &.$- / r \cdot \xi$ & $\cdot \cdot / M F^{* \prime}$ &.$- / 9 \mathrm{vq}^{* * *}$ & / & يتانسيل اسمزى \\
\hline . $/ \pi+9$ &.$/ 191$ & $\%$ & . &.$/ .4$ & . & $-/ / \cdot \cdot \lambda$ & .1 .91 & $-\% / \Delta t$ & $-\cdot / \cdot A r$ &.$/ \mathrm{Tr}$ & .1 .90 & . $/ 2 \Delta t^{* * *}$ & 1 & . &.$- / .19$ & 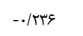 &.$- / . / r$ &.$- / .44$ & .//MS &.$- / \% \Delta q$ & ..$w$ & الرفناع بوته \\
\hline . &.$/ \mathrm{rv}$. & $-\cdot / 1 . r$ & $\cdot / \pi r r$ & 政 & $\cdot / r v$ & $\cdot 1+\oplus_{\Delta}$ &.$/ 19$. & $\cdot 1 .+v$ & $\%$ & 1.91 & $\cdot r \cdot$. & 1 &.$/ 991 \mathrm{v}$ & .11 .9 &.$- / .91$ & $-\cdot / T / \varphi$ & $\cdots / M$ & $-\cdots / r_{\Delta}$ & $\because / 109$ &.$- / \pi T r$ & 1.9r & وزن خشك بوته \\
\hline$. / v \cdot .^{* * *}$ & . Nws" & $\cdot / \triangle A A^{\prime \prime *}$ &.$/ 190^{* * *}$ & . $/ \wedge q^{* * *}$ &.$/ a r t^{40}$ & ./a1r" &.$/ 99 v^{\prime \prime}$ & $.190)^{* * *}$ &.$/ A \& \xi^{* *}$ & . /rap & 1 & $-\cdot / r \cdot v$ & -./TH & $-\cdot / \mathrm{NVY}{ }^{* *}$ & $\cdot \mid A N A^{" \prime}$ &.$/ 9940$ &.$- / \Gamma \cdot 1$ & . $/ \mathrm{ver}$ & $. / 20 . . " *$ & $\cdot|\Lambda| r^{* *}$ & $-\cdot / A F q^{\mu *}$ & طول سنبله \\
\hline.$/ K I$ & $.1 . .9$ &.$- / r \cdot r$ & . & . IFY & r rer & / RVY & .rar & $. / r \Lambda \mid$ & $. / 4 . q$ & 1 & $-\ldots / F r s$ & / RTV &.$|M|$ &. $\mid F A V^{*}$ &.$- / 4 \% 98^{\circ}$ & 更 &.$/$. & ../KY & - & $-\cdot \Delta \Delta \Delta^{*}$ & . & تهاد ريشه در بوته \\
\hline$\cdot / \Delta v \Delta \theta^{* * *}$ & $.19 \times w^{* 1}$ &.$- / \mathrm{wq}^{\circ *}$ & .(va $)^{\text {** }}$ & $\cdot / \lambda \cdot F^{* * *}$ & . $/ A r r^{* \prime}$ &.$/ 9 \cdot 1^{* *}$ & . $/ 4 r 9 "$ &.$/ 9 \Lambda^{* * *}$ & 1 & $-\cdot / \Delta \Delta S^{*}$ & $. / 9 . r^{* "}$ & - $-/ /$ Ff &.$- / / T V$ & $-\cdot / / \mathrm{AFF} \mathrm{F}^{* *}$ & $\cdot / \mathrm{APa} "$ &.$/ 1940$ &.$- / . \psi$ &.$/ T r$ & . &.$/ 9 \mathrm{~V}{ }^{* * *}$ & $-\cdot / \Delta V^{\prime \prime}$ & طول ريشه \\
\hline $.19 \cdot r^{* * *}$ & $\cdot|\Lambda| v^{* *}$ &.$- /$ ar..$"$ & $\cdot \mid \Delta \Delta N^{* * *}$ & $. / 9 . q^{* * *}$ & . INV" & ./Arso" & . & 1 & . $/ 4 V^{\prime \prime \prime}$ & - & . $/ \mathrm{NPF}^{\circ "}$ & - & $-\cdot / \| r$ & $-\cdot / \Delta r f^{2 *}$ & $\cdot \mid A N r^{* \prime}$ & $\cdot \mid A v \cdot{ }^{. " *}$ & $-.1 .9 \Lambda$ & . & . $/ \mathrm{Arq}{ }^{a * *}$ &. $\mid F \Delta F^{* * *}$ & $-\cdot / N \varepsilon^{4 * *}$ & حجم ريشه \\
\hline.$N \cdot r^{*}$ & $\cdot M M^{* *}$ & $-\left.\cdot|N|\right|^{* *}$ &.$/ 199 "$ & $.19 \cdot 0.0 *$ & .ar." & $\cdot 1 / 99 . "$ & , & 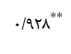 & . $9999^{\circ "}$ & 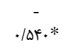 & 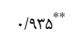 & $--/ 1 \cdot 9$ & $-\cdot / 1 \cdot r$ & $-\sigma / \Lambda r^{*}$ & $\cdot|\Delta r\rangle^{* \prime}$ & . $/$ arr" & $-1 / .09$ &.$(F+1$ & . $/ M r^{\prime \prime *}$ &. Mro" &.$- / M r^{\prime \prime}$ & وزن خشك ريشه \\
\hline. $\mid 91.0^{* * *}$ & $\cdot \mid \lambda \cdot r^{* *}$ & -./va & $\cdot(\lambda \cdot)^{* * *}$ & $\cdot / \mu r^{* * *}$ & $\cdot \mid A r{ }^{\prime \prime}$ & ' & $\cdot \mid \Lambda \cdot r^{* * *}$ & $\cdot \mid A s s^{* *}$ & . $/$ Affe" & 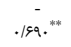 & . & $-\cdot / 1 \cdot \Delta$ &.$- / . \Delta f$ & $-. / 919 . *$ & $.19980 "$ & . & $-(\cdot . \Delta)$ & $\cdot / r \cdot r$ & $\cdot \mid A \Delta D^{\prime \prime *}$ &. $\mid 599^{9 * *}$ & $-. / 1 / 10^{4 *}$ & قطر ريشه \\
\hline $.199 . * *$ & $.199 y^{* *}$ & $-\cdot / \Delta r \gamma^{\theta *}$ & $\cdot \operatorname{sag} 9^{* * *}$ & $. / 9 \times D^{* * *}$ & 1 & $\cdot \mid / \cdot \Lambda^{* *}$ &.$/ 29 a^{\circ "}$ & $\cdot / \wedge 9 T^{* * *}$ & .ar." & $--/ / 4 q 9$ &.$/ 995^{\circ *}$ & $-\because / \wedge \Delta$ & $-\cdot / r \cdot r$ & $-\cdot|A V|^{* *}$ & $\cdot \mid A v y^{" \prime}$ & $. / 99)^{10}$ & -./49 & . &. $\mid A+\Delta \Delta^{* * *}$ & . $/$ vav"* & $-\cdot / \Delta \Delta V^{\prime \prime \prime}$ & تعداد ينجه در بوته \\
\hline. $\mid \phi \varphi r^{* * *}$ & $.19 \times 0^{* 1 *}$ & $-. / 199)^{\prime *}$ & $\cdot \mathrm{MM}^{* *}$ & 1 & . & $\cdot 1 / .9 \cdot q^{* *}$ &.$/ 24 \Lambda^{\circ "}$ & $\cdot / \wedge 9 T^{* * *}$ &.$/ 91 F^{* "}$ &.$-|k| s$ & . & - $-/ \wedge Y$ & $-\cdot / r \cdot \Delta$ & $-\cdot|A V\rangle^{* *}$ & $\cdot / \mathrm{NW}^{\prime \prime}$ &.$/ 991^{\prime *}$ & -./19y & . & $.194 \Lambda^{\prime \prime *}$ & $\cdot / \Lambda \cdot r^{* * *}$ & $-\cdot / A F F^{* *}$ & تعداد بنجه باور در بوته \\
\hline$\cdot M F^{* *}$ &.$M N^{* *}$ & $-\cdot / \wedge r \Delta \Delta^{* *}$ & , &.$/ 99 N^{* * *}$ & .av." & $\cdot \mid M r r^{* *}$ & . &.$/ 91 r^{* * *}$ & . $/ 9 \mathrm{ros}$ & - &.$|a v|^{* *}$ & $-\cdot / r \cdot r$ & $-\cdot / r \cdot r$ & $-\cdot|A V|^{* *}$ & $\cdot \mid M . "$ & . $/$ avi" & $-\cdot / \Delta \mathrm{Dr}$ &.$/ 499^{\circ}$ &.$/ a r T^{n+*}$ & $\cdot|\wedge\rangle^{* * *}$ & $-\cdot / \Delta A^{* * *}$ & تعداد سنبله در بوته \\
\hline$-\cdot / \Delta \mid r^{*}$ & $-\cdot / v a r^{n+\infty}$ & , &.$- \mid k s 8^{*}$ & $-. / 4+\%$ & -./4 &.$- / r a r$ &.$- / r \Delta r$ &.$- / r Y \wedge$ &.$- / r 19$ & $\cdot|\cdot \lambda|$ & . Frs & $-\cdot / \cdot r)$ &.$- / .14$ & $\cdot / \pi r$ &.$-|+9|$ &.$- / A F F^{\circ}$ & .rif & $-. / \%+\lambda$ &.$- / \% \Delta \Lambda^{*}$ &.$- / \% v^{*}$ & . & تعناد دانه در سنبله \\
\hline $.19 .1^{* *}$ & ' &.$- / / 998$ &.$* \% \mathrm{~V}$ &.$|F Y|^{\circ}$ & . Fes & $.1 \% a 9$ & . Ks99 & 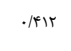 & . rvv. &.$- / 154$ & $\cdot \mid F \Delta F^{*}$ & - &.$- / k r r$ &.$- / \pi T r$ & 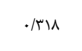 & . $\mid \Leftrightarrow 9 \Lambda^{*}$ & $|\Delta| \Delta \Delta^{*}$ &.$/ 10 \mathrm{~A}$ &.$|F v|^{*}$ & $\left.|| F r\right|^{* * *}$ &.$- / \pi r$ & وزن هزار دانه \\
\hline , &. $\mid \Delta s F^{* \prime}$ & -.//9QD &.$/ A \Delta F^{* * *}$ & $. / a r y=$ & . & $\cdot \mid A T A^{*}$ & . $/ 9 \cdot 9^{\circ *}$ &.$/ 9 \mathrm{NA}^{* * *}$ & 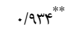 &.$- / \Delta r \Delta \Delta^{*}$ &.$/ 440^{\circ "}$ &.$- / r \cdot \lambda$ & 年/ &.$- / A 4 r^{* * *}$ & $\cdot|A|^{\prime \prime "}$ & . $/$ arfe" & $-\because / \lambda$. & 的 & . $/ 9 \cdot \mathrm{C}^{* * *}$ & $\cdot|\Lambda| F v$ & $-* / A r q^{* *}$ & عملكرد دانه \\
\hline
\end{tabular}



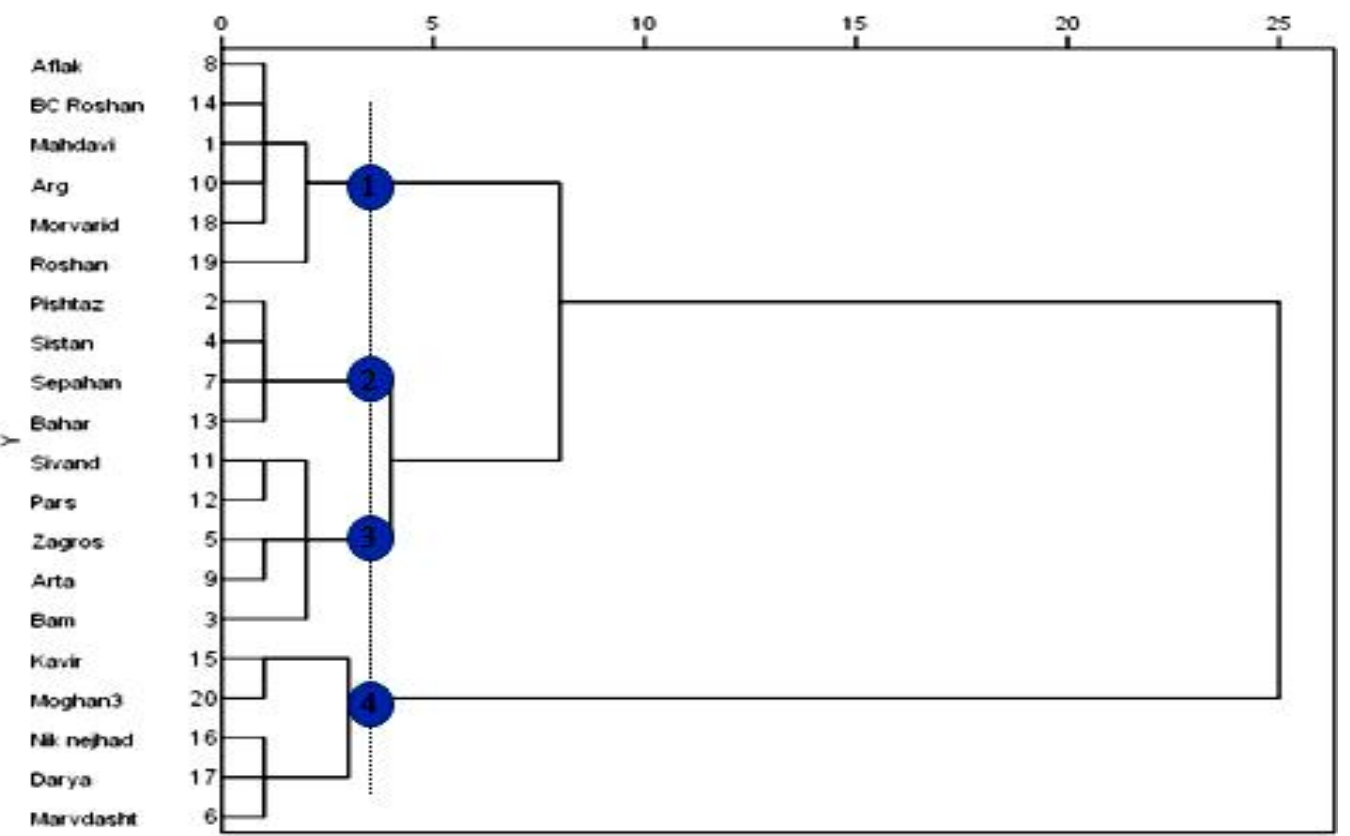

شكل r- دندروگرام ارقام گَندم بهاره براساس همه صفات مورد مطالعه با استفاده از روش Ward و برش توسط تابع تشخيص تحت شرايط شاهد

Figure 2. Dendrogram of spring wheat cultivars based on studied traits using Ward's method and cutting by discriminant function under control conditions

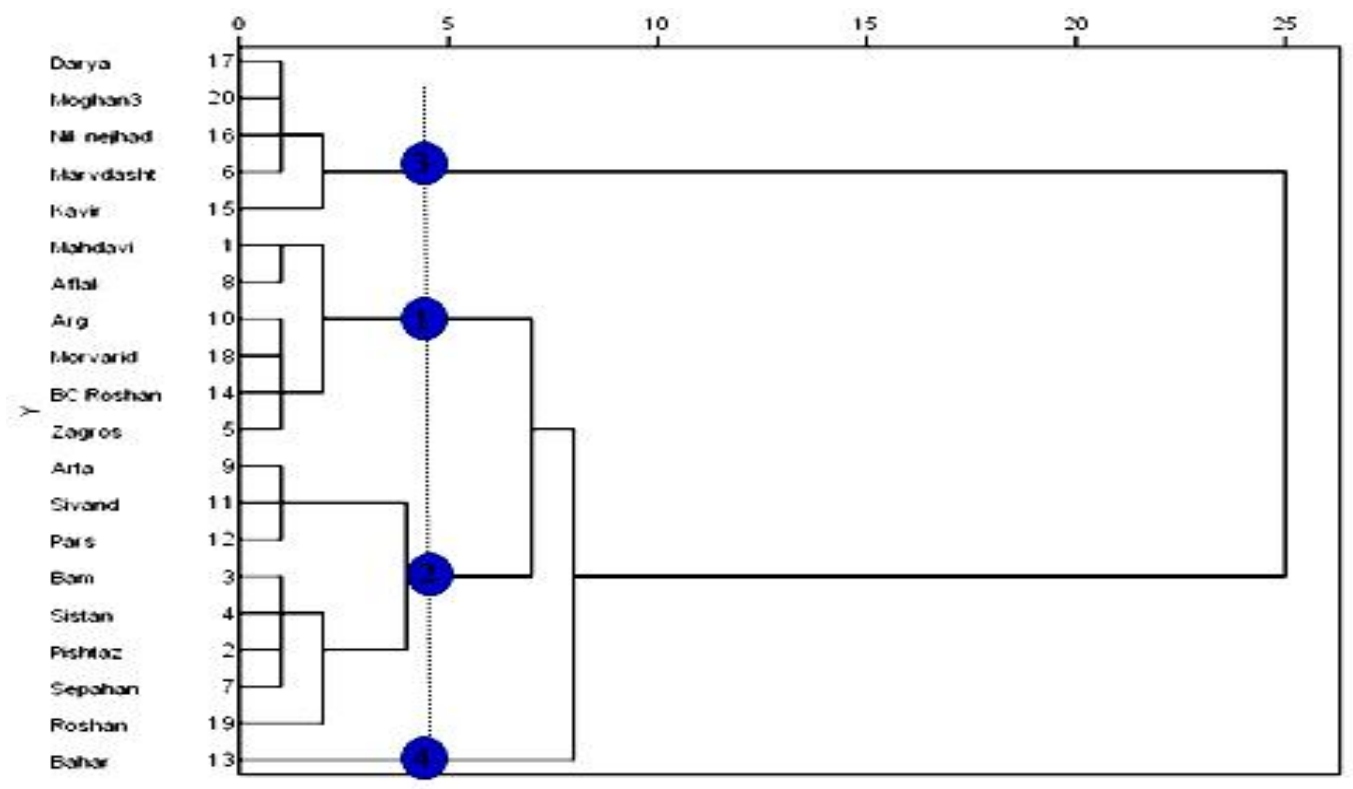

شكل س- دندروكرام ارقام كندم بهاره بر اساس صفات مورد مطالعه با استفاده از روش Ward و برش توسط تابع تشخيص تحت شرايط تنش خشكى

Figure 3. Dendrogram of spring wheat cultivars based on studied traits using Ward's method and cutting by discriminant function under stress conditions 
1. Araus, JL T. Amaro, T. Voltas, J. Nakkoul and MM. Nachit 1998. Chlorophyll fluorescence selection criterion for grain yield in durum wheat under Mediterranean conditions. Field Crops Research, 55: 209-223.

2. Araus, J.L., S. Ceccarelli and S. Grando. 1997. Relationship between leaf structure and carbon isotope discrimination in field-grown barley. Plant Physiology and Biochemistry, 35: 533-541.

3. Arias, D. 2007. Calibration of LAI-2000 to Estimate Leaf Area Index and Assement of its Relationship with stand productivity in six Native and Introduced tree Species in costarica. Forest Ecology and management, 247: 85-193.

4. Bajji, M., S. Lutts and K.M. Kinet. 2001. Water deficit effects on solute contribution to osmotic adjustment as a function of leaf ageing in three durum wheat (Triticum durum Desf.) cultivars performing in arid conditions. Plant Science, 60: 669-681.

5. Baker, N.R. and E. Rosenquist. 2004. Application of chlorophyll fluorescence can improve crop production strategies: An examination of futurepossibilities. Journal of Experimental Botany, 55: 1607-1627.

6. Basal, H., P. Bebeli, C.W. Smith and P. Thyton. 2003. Root growth parameters of converted racestocks of upland cotton and two $\mathrm{BC}_{2} \mathrm{~F}_{2}$ populations. Crop Science, 43: 1983-1988.

7. Blum, A. 1996. Crop responses to drought and the interpretation of adaptation. Journal of Plant Growth Regulation, 20: 135-148.

8. Blum, A. 2005. Drought resistance, water-use efficiency, and yield potential, are they compatible, dissonant, or mutually exclusive. Australian Journal of Agricultural Research, 56: 1159-1168.

9. Chen, T.H.H. and N. Murata. 2002. Enhancement of tolerance of abiotic stress by metabolic engineering of betaines and other compatible solutes. Current Opinion in Plant Biology, 5: 250-257.

10. Chimenti, C.A., J. Pearson and A.J. Hall. 2002. Osmotic adjustment in Maize: Genetic variation and association with water uptake. In: Edmeades, G.O. (ed.). Developing Drought and Low N-Tolerant Maize. 200-203 pp., Centro Internacional de Mejoramiento de Maiz y Trigo (CIMMYT), Mexico DF (Mexico). CIMMYT.

11. Edmeads, G.O., J. Bolanos, H.R. Laffite, S. Rajaram, W. Preffer, and R.A. Fisher. 1989. Traditional approaches to breeding for drought resistance in cereals. CABI, 52pp.

12. Gupta, N.K., S. Gupta and A. Kumar. 2001. Effect of water stress on physiological attributes and their relationship with growth and yield of wheat cultivars at different stages. Journal of Agronomy and Crop Science, 186: 55-58.

13. Hasegawa, P.M., R.A. Bressan, J.K. Zhu and H.J. Bohnert. 2000. Plant cellular and molecular responses to high salinity. Annual Review of Plant Physiology and Plant Molecular Biology, 51: 463499.

14. Heyne, E.G. 1987. Wheat and wheat improvement. $2^{\text {nd }}$ edn. American Society of Agronomy, Madison, Wisconsin, USA.15 pp.

15. Inamullah, Z., A. Swati, A. Latif and M. Siraj-u-Din. 1999. Evaluation of lines for drought tolerance in wheat (Triticum aestivum L.). Scientific Khyber, 12: 39-48.

16. Jafarzadeh, A.A., R. Kasraii and M.R. Neishabouri. 1997. Detailed studies of 18 acres of land, soil research station Karkaj. Iranian Journal of Agricultural Sciences, 7: 187-213 (In Persian).

17. Jones, H.G. 1983. Plants and microclimate: A quantitative approach to environmental plant physiology. Camb, University Press, Camb. London, 54 pp.

18. Kavi Kishor, P.B., S. Sangam, R.N. Amrutha, P.S. Laxmi, K.R. Naido, S.S. Rao, K. Reddy, P. Theriappan, and N. Sreenivasulu. 2005. Regulation of proline biosynthesis, degradation, uptake and transport in higher plants: Its implications in plant growth and abiotic stress tolerance. Current Science, 88: 424-438

19. Khan, A.J., F. Azam, A. Ali, M. Tariq and M. Amin. 2005. Inter-relationship and path co-efficient analysis for biometric traits in drought tolerant wheat (Triticum aestivum L.). Asian Journal of Plant Sciences, 4: 540-543.

20. Khanzada Barkat, S., A. Ala, M. Yasin, M.U. Ashraf, S.M. Shirazi, R. Alam, M. Ansari, M. Ali, A. Mukhtiar, S.M. Mujataba and M.A. Khan. 2001. Effect of water stress on yield and yield components of different Guar (Cyamopsis tetragonaloba L.) genotypes. Pakistan Journal of Biological Sciences, 4: 371-374.

21. Kramer, P.J. 1969. Plant and soil water relationships. Modem synthesis. Mc Graw-Hill Book co, New York. 84pp.

22. Kumar, A. and D.P. Singh. 1998. Use of physiological indices as a screening technique for drought tolerance in oilseed Brassica species. Annals of Botany, 81: 413-420.

23. Kutlu, I. and G. Kinaci. 2010. Evaluation of drought resistance indicates for yield and its components in three Triticale Cultivars. Journal of Tekirdag Agriculture Faculty, 7: 95-103.

24. Long, S.P., S. Humphries and P.G. Falkowski. 1994. Photo inhibition of photosynthesis in nature. Annual Review of Plant Physiology and Plant Molecular Biology, 45: 633-662.

25. Lu, Z. and P.M. Neumann. 1999. Low cell-wall extensibility can limit maximum leaf growth rates in rice. Crop Science, 39: 126-130.

26. Maroco, J.P., M.L. Rodriges, C. Lopes and M.M. Chaves. 2002. Limitaion to leaf photosynthesis in grapevine under drought metabolic and modeling approaches. Functional Plant Physiology, 29: 1-9.

27. Martinez, J.P., S. Luttus, A. Schanck and M. Banjji. 2004. Is osmotic adjustment required for water stress resistance in the Mediterranean shrub Atriplexhalimus L. Journal of Plant Physiology, 16: 10411051. 
28. Mc Manus, M.T., R.L. Bieleski, L.R. Caradus and D.J. Barker. 2000. Pinitoal accumulation in mature leaves of white clover in response to a water deficit. Environmental and Experimental Botany, 43: 1118.

29. Moghaddam, M., M. Basirat, F. Rahimzadeye Khoii and M.R. Shakiba. 1993. Path analysis for seed yield, its components and some morphological traits in winter wheat. Iranian Journal of Agricultural Sciences, 4: 48-73 (In Persian).

30. Monneveux, P. and E. Belhassen. 1996. The diversity of drought adaptation in the wide. Plant Growth Regulation, 20: 85-92.

31. Morant-Manceau, A., E. Pradier and G. Tremblin. 2004. Osmotic adjustment, gas exchanges and chlorophyll fluorescence of a hexaploid triticale and its parental species salt stress. Journal of Plant Physiology, 169: 25-33.

32. Musick, J.T., O.R. Jones, B.A. Stewart and D.A. Dusek. 1994. Water-yield Relationships for Irrigated and Dryland Wheat in the U.S. Southern Plains. Agronomy Journal, 86: 980- 986.

33. Noroozi, M. and S.A.R. Kazemini. 2012. Effect of Water Stress and Plant Density on Growth and Seed Yield of Safflower. Iranian Journal of Field Crops Research, 10: 781-788 (In Persian).

34. Ozturk, L. and Y. Demir. 2002. In vivo and in vitro protective role of proline. Plant Growth Regulation, 38: 259-264.

35. Paleg, L.G. and D. Aspinall. 1981. The physiology and biochemistry of drought resistance in plants. Academic press, Cambridge, Massachusetts, United States, $492 \mathrm{pp}$.

36. Passioura, J.B. 1997. Grain yield, harvest index and water use of wheat. Australian Institute of Agricultural Science, 43: 117-120.

37. Qadir, G.S. Mohammad and C. Mumtaz Akhtar. 1999. Effect of water stress on growth and yield performance of four wheat cultivars. Pak. Journal of Biological Sciences, 2: 236-239.

38. Reynolds, M.P., M. Balota, M.I.B. Delgado, I. Amani and R.A. Fischer. 1994. Physiological and morphological traits associated with spring wheat yield under hot, dry irrigated conditions. Australian Journal of Plant Physiology, 21: 717-730.

39. Safarnejad, A. 2004. Chractrization of somaclones of alfalfa (Medicago sativa L.) for drought tolerance. Journal of Agricultural Science andTechnology, 6: 121-127.

40. Sairam, R.K., P.S. Deshmukh and D.S. Shukla. 1997. Tolerance of drought and temperature stress in relation to increased antioxidant enzyme activity in wheat. Journal of Agronomy and Crop Science, 178: $171-178$.

41. Saleem, M. 2003. Response of durum and bread wheat genotypes to drought stress: Biomass and yield components. Asian Journal of Plant Sciences, 2: 290-293.

42. Serraj, R. and T.R. Sinclair. 2002. Osmolyte accumulation: can it really help increase crop yield under drought condition. Plant, cell and Environment, 25: 333-341.

43. Schonfeld, M.A., R.C. Johnson, B.F. Carver and D.W. Mornhinweg. 1988. Water relations in winter wheat as drought resistance indicators. Crop Science, 28: 526-531.

44. Sharma, R.B. and B.P. Ghildyal. 1977. Soil water-root relations in wheat. Water extraction rate of wheat roots that develop under dry and moist conditions. Agronomy Journal, 69: 231-233.

45. Sharp, R.E., G.L. Davis, G.K. Sprivinger, D.P. Schachtman and J. Gros. 2004. Root growth maintenance during water deficit: Physiology to functional genomics. Journal of Experimental Botany, 55: 2343-2351.

46. Sinclair, T. and M. Ludlow. 1985. Who taught plants thermodynamics? The unfulfilled potential of plant water potential. Australian Journal of Plant Physiology, 12: 213- 217.

47. Singh, M., J.P. Srivastava and A. Kumar. 1990. Effects of water on water potential components in wheat genotypes. Indian Journal of Plant Physiology, 33: 312-317.

48. Tan, B.H. and G.M. Hollaran. 1982. Variation and collerations of proline accumulation in spring wheat cultivars. Crop science, 22: 459-462.

49. Toorchi, M., H.E. Shashidhar, N. Sharma, and S. Hittalmani. 2002. Tagging QTL for maximum root length in rainfed lowland rice (Oryza sativa L.) using molecular markers. Cellular and Molecular Biology Latters, 7: 771-776.

50. Usman Khan, M., M.I. Chowdhry, I. Khalig, and R. Ahmad. 2003. Morphological response of various genotypes to drought conditions. Asian Journal of Plant Sciences, 2: 392-394.

51. Vendruscolo, A.C.G., I. Schuster, M. Pleggi, C.A. Scapim, H.B.C. Molinari, C.J. Marar and G.C. Vieria. 2007. Stress-induced synthesis of proline confers tolerance to water deficit in transgenetic wheat. Journal of Plant Physiology, 164: 1367-376.

52. Wange, W., B. Inocur and A. Altman. 2003. Plant responses to drought, salinity and extreme temperature: towards genetic engineering for stress tolerance. Planta, 218: 1-14.

53. Winter, S.R., J.T. Musick and K.B. Porter. 1988. Evaluations of screening techniques for breeding drought-resistant winter wheat. Crop Science, 28: 512-516.

54. Xue, Q., Z. Zhu, J.T. Musick, B.A. Stewart and D.A. Dusek. 2003. Root growth and water uptake in winter wheat under deficit irrigation. Plant and Soil, 257: 151-161.

55. Zheng, Y., A. Zhu and L. Tian. 2000. Changes of proline levels and abscisic acid content in tolerantsensitive cultivars of soybean under osmotic conditions. Www.soygenetics.orglarticles/sgn 200011.htm.

56. Zlatev, Z. and I.T. Yordanov. 2004. Effect of soil drought on photosynthesis and chlorophyll fluorescence in bean plants. Bulgarian Journal of Plant Physiology, 30: 3-18. 


\title{
Evaluation of Spring wheat Cultivars for Physiological, Morphological and Agronomic Traits under Drought Stress
}

\author{
Mohammad Reza Naghavi ${ }^{1}$, Mohammad Moghaddam² ${ }^{2}$, Mahmoud Toorchi ${ }^{2}$ and \\ Mohammad Reza Shakiba ${ }^{2}$ \\ 1- Assistant Professor, Department of Agriculture, Payame Noor University, Iran \\ (Corresponding author: mr_naghavi@ymail.com) \\ 2- Professor, University of Tabriz \\ Received: April 11, $2014 \quad$ Accepted: September 15, 2014
}

\begin{abstract}
For evaluation of the reaction twenty of spring wheat cultivars under complete irrigation (control) and drought stress condition starting before booting stage, an experiment was conducted in split plot basis of Randomized Complete Block Design with 4 replications in two cropping years (2010-2011 and 2011-2012) in research farm of Agriculture Faculty, University of Tabriz. The results of variance analysis of traits were showed significant difference between stress levels and between cultivars for all studied traits. Stress cause to significantly decrease of grain yield, yield components, plant height and other morphological traits. Also, values of physiological traits have decreased under drought stress and this decrease was not similar for all cultivars. Further, quantitative amounts of root traits were increased for plant defense apposite to drought stress and this increase was more in tolerant cultivars than others. On the other hand, assessment of correlation coefficients between traits was showed that grain yield had the highest positive and significant correlation with number of spike per plant under control and drought stress conditions. Also, dendrogram of cluster analysis grouped cultivars into four groups under both of control and drought stress conditions. According to achieved results of mean comparisons and cluster analysis, Kavir, Niknejhad, Darya, Moghan3 and Marvdasht were the most tolerant cultivars and Bahar was the most sensitive cultivar. So, it seems that these tolerant cultivars are suitable for direct culture in farm that has limitation for irrigation during end of growth period.
\end{abstract}

Keywords: Cluster analysis, Drought stress, Drought tolerant, Spring wheat 